\title{
Geometry of holomorphic mappings and Hölder continuity of the pluricomplex Green function
}

\section{Rafał Pierzchała ${ }^{1}$}

Received: 12 September 2019 / Revised: 15 January 2020 / Published online: 12 February 2020

(c) The Author(s) 2020

\section{Abstract}

We provide a solution to a long-standing open problem that lives in the interface of pluripotential theory and multivariate approximation theory. The problem is to characterize the holomorphic maps which preserve Hölder continuity of the pluricomplex Green function associated with a compact subset of $\mathbb{C}^{N}$. We also prove, under mild restrictions, that nondegenerate holomorphic maps preserve Markov's inequality for polynomials.

\section{Introduction}

For each compact set $\emptyset \neq K \subset \mathbb{C}^{N}$, the following function

$$
V_{K}(z):=\sup \left\{\phi(z): \phi \in \mathcal{L}\left(\mathbb{C}^{N}\right), \phi \leq 0 \text { on } K\right\}
$$

$\left(z \in \mathbb{C}^{N}\right)$, where $\mathcal{L}\left(\mathbb{C}^{N}\right)$ denotes the class of plurisubharmonic functions $\phi$ in $\mathbb{C}^{N}$ (see [23] for the definition and basic properties of plurisubharmonic functions) satisfying the logarithmic growth condition

$$
\sup _{z \in \mathbb{C}^{N}}[\phi(z)-\log (1+|z|)]<+\infty
$$

Dedicated to the memory of Professor Józef Siciak.

\section{Communicated by Ngaiming Mok.}

Rafał Pierzchała

Rafal.Pierzchala@im.uj.edu.pl

1 Faculty of Mathematics and Computer Science, Jagiellonian University, ul. Łojasiewicza 6, 30-348 Kraków, Poland 
is called the pluricomplex Green function of $K$ (with pole at infinity) or the Siciak-Zakharyuta extremal function; see for example $[4,23,25,39,43,44]$ and the bibliography therein. Here and subsequently, | | denotes the maximum norm in $\mathbb{C}^{N}$.

If $N=1, K \subset \mathbb{C}$ is nonpolar (that is, of positive logarithmic capacity), and $K_{\infty}$ denotes the unbounded component of $\overline{\mathbb{C}} \backslash K$, then $V_{K}$ is harmonic in $\mathbb{C} \backslash K$ and the restriction of $V_{K}$ to $K_{\infty}$ is the Green function of $K_{\infty}$ with pole at infinity; see [44, 7.1 and 7.2].

Definition 1.1 (see [23]) A set $A \subset \mathbb{C}^{N}$ is said to be pluripolar if, for each point $a \in A$, there exists an open neighbourhood $U$ of $a$ such that $A \cap U \subset\{z \in U: \varphi(z)=-\infty\}$ for some plurisubharmonic function $\varphi: U \rightarrow[-\infty,+\infty)$.

If $K \subset \mathbb{C}^{N}$ is a nonpluripolar compact set, then the upper semicontinuous regularization $V_{K}^{*}(z):=\limsup _{\zeta \rightarrow z} V_{K}(\zeta)$ of $V_{K}$ is plurisubharmonic in $\mathbb{C}^{N}$ and satisfies the complex Monge-Ampère equation:

$$
\left(d d^{c} V_{K}^{*}\right)^{N}=0 \quad \text { in } \mathbb{C}^{N} \backslash K
$$

see $[4,23,26]$ for more details. Moreover, for each compact set $\emptyset \neq K \subset \mathbb{C}^{N}$,

$$
K \text { is pluripolar } \Longleftrightarrow V_{K}^{*} \equiv+\infty \Longleftrightarrow V_{K}^{*} \notin \mathcal{L}\left(\mathbb{C}^{N}\right) \text {; }
$$

see [44, Corollary 3.9 and Theorem 3.10]).

We should also recall that the pluricomplex Green function is essentially equivalent to the Siciak extremal function. The latter is defined by the formula

$\Phi_{K}(z):=\sup \left\{|Q(z)|^{1 / \operatorname{deg} Q}: Q \in \mathbb{C}\left[z_{1}, \ldots, z_{N}\right], \operatorname{deg} Q>0\right.$ and $\left.\|Q\|_{K} \leq 1\right\}$

$\left(z \in \mathbb{C}^{N}\right)$, where $\|Q\|_{K}:=\sup _{z \in K}|Q(z)|$. Strictly speaking, for each compact set $\emptyset \neq K \subset \mathbb{C}^{N}$,

$$
V_{K}=\log \Phi_{K}
$$

see [23, Theorem 5.1.7].

It may be worth noting that, for each $Q \in \mathbb{C}\left[z_{1}, \ldots, z_{N}\right]$ with $\|Q\|_{K}>0$ and each $z \in \mathbb{C}^{N}$

$$
|Q(z)| \leq\left(\Phi_{K}(z)\right)^{\operatorname{deg} Q}\|Q\|_{K}
$$

(We adopt here the convention that, for each $r \geq 0,(+\infty)^{r}:=+\infty$. Moreover, for $K$ being nonpluripolar, the additional assumption that $\|Q\|_{K}>0$ is superfluous.) This trivial (but useful) estimate is called the Bernstein-Walsh inequality.

The pluricomplex Green function has been used to study various problems in (real and complex) analysis, functional analysis, pluripotential theory, complex dynamics and in approximation theory. From the point of view of applications, the most desirable property of this function is the HCP property. 
Definition 1.2 (see [29]) We say that a compact set $\emptyset \neq K \subset \mathbb{C}^{N}$ has the HCP property if there exist $\varpi, \mu>0$ such that, for each $z \in K_{(1)}$,

$$
V_{K}(z) \leq \varpi(\operatorname{dist}(z, K))^{\mu} .
$$

In the above definition, and subsequently, we use the following notation: for each set $\emptyset \neq A \subset \mathbb{C}^{N}$ and each $r>0$, we put

$$
A_{(r)}:=\left\{z \in \mathbb{C}^{N}: \operatorname{dist}(z, A) \leq r\right\}
$$

Since $V_{K} \equiv 0$ in $K$, the HCP property can be reformulated in the following way: for each $z \in K_{(1)}$ and each $z^{\prime} \in K$,

$$
\left|V_{K}(z)-V_{K}\left(z^{\prime}\right)\right| \leq \varpi\left|z-z^{\prime}\right|^{\mu}
$$

Surprisingly, a simple argument due to Błocki shows that this estimate already implies Hölder continuity of $V_{K}$ in $\mathbb{C}^{N}$; cf. [46, Proposition 3.5] or [42, Lemma 2.3]. More precisely, for each $\mu>0$, the following two conditions are equivalent:

- There exists $\varpi>0$ such that (1.4) holds.

- There exists $\tilde{\omega}>0$ such that, for all $z, z^{\prime} \in \mathbb{C}^{N}$,

$$
\left|V_{K}(z)-V_{K}\left(z^{\prime}\right)\right| \leq \tilde{\varpi}\left|z-z^{\prime}\right|^{\mu}
$$

Example 1.3 Assume that $K_{1}, \ldots, K_{N}$ are nonempty compact subsets of $\mathbb{C}$ such that, for each $j \leq N$ and each connected component $E_{j}$ of $K_{j}$, we have $\operatorname{diam}\left(E_{j}\right) \geq \eta$, the constant $\eta>0$ being independent of $j$. Set $K:=K_{1} \times \cdots \times K_{N}$. By [45, Lemma 3.1], for each $j \leq N$ and each $u \in\left(K_{j}\right)_{(1)}$,

$$
V_{K_{j}}(u) \leq \varpi \sqrt{\operatorname{dist}\left(u, K_{j}\right)},
$$

where

$$
\varpi:=\frac{4}{\eta}\left(1+\sqrt{1+\frac{\eta}{2}}\right) .
$$

Fix $z=\left(z_{1}, \ldots, z_{N}\right) \in K_{(1)}$. By [23, Theorem 5.1.8],

$$
\begin{aligned}
V_{K}(z) & =\max \left\{V_{K_{1}}\left(z_{1}\right), \ldots, V_{K_{N}}\left(z_{N}\right)\right\} \\
& \leq \varpi \max \left\{\sqrt{\operatorname{dist}\left(z_{1}, K_{1}\right)}, \ldots, \sqrt{\operatorname{dist}\left(z_{N}, K_{N}\right)}\right\} \\
& =\varpi \sqrt{\operatorname{dist}(z, K)},
\end{aligned}
$$

which yields the HCP property for the set $K$, with the exponent $\mu=1 / 2$. Let us emphasize that, for some product sets of planar compact sets, this exponent is not the best possible. For example, for a polydisk 


$$
\mathbb{D}(a, r):=\left\{z \in \mathbb{C}^{N}:|z-a| \leq r\right\}
$$

( $a \in \mathbb{C}^{N}, r>0$ ), the HCP property holds with the exponent $\mu=1$. Indeed, since

$$
V_{\mathbb{D}(a, r)}(z)=\max \left\{0, \log \frac{|z-a|}{r}\right\}
$$

(see [23, Example 5.1.1]), it follows that

$$
V_{\mathbb{D}(a, r)}(z) \leq \frac{\operatorname{dist}(z, \mathbb{D}(a, r))}{r}
$$

for all $z \in \mathbb{C}^{N}$. On the other hand, however, for the cube $[-1,1]^{N} \subset \mathbb{R}^{N} \subset \mathbb{C}^{N}$, the exponent $\mu=1 / 2$ is the best possible, which follows from the formula

$$
V_{[-1,1]^{N}}(z)=\max \left\{\log \left|z_{1}+\sqrt{z_{1}^{2}-1}\right|, \ldots, \log \left|z_{N}+\sqrt{z_{N}^{2}-1}\right|\right\},
$$

valid for all $z=\left(z_{1}, \ldots, z_{N}\right) \in \mathbb{C}^{N}$; see [23, Corollary 5.4.5]. For each $j \leq N$, the square root is so chosen that $\left|z_{j}+\sqrt{z_{j}^{2}-1}\right| \geq 1$.

There have been several significant advances in understanding the HCP property for compact subsets of $\mathbb{C}$. In particular, we should mention here a very interesting work of Carleson and Totik [16], in which they give a sufficient Wiener-type criterion for a compact set $K \subset \mathbb{C}$ to have the HCP property.

Incomparably less has been done so far in the multivariate case (that is, for $N>1$ ). However, Pawłucki and Pleśniak in a seminal paper [29] give a sufficient geometric condition (UPC condition) for a compact set $K \subset \mathbb{R}^{N}$ to have the HCP property. Furthermore, in [29,30,32] large and natural classes of compact sets in $\mathbb{R}^{N}$ satisfying the UPC condition (and hence with the HCP property) are provided. More precisely, these classes consist of all compact, fat (a set $E$ is said to be fat if $\bar{E}=\overline{\operatorname{Int} E}$ ) and definable sets in certain o-minimal structures; see [18] for the definition of an ominimal structure. Each compact, fat and semianalytic subset of $\mathbb{R}^{N}$ is an explicit example of such a set.

Definition 1.4 (see [5,27]) Let $\Omega \subset \mathbb{R}^{N}$ be an open set. A set $A \subset \Omega$ is said to be a semianalytic subset of $\Omega$ if, for each point in $\Omega$, we can find a neighbourhood $W$ such that $A \cap W$ is a finite union of sets of the form

$$
\left\{x \in W: \xi(x)=0, \xi_{1}(x)>0, \ldots, \xi_{m}(x)>0\right\},
$$

where $\xi, \xi_{1}, \ldots, \xi_{m}$ are real analytic functions in $W$.

One of the long-standing open problems concerning the HCP property is the following.

Problem 1.5 (Pleśniak, 1988) Let $h: U \rightarrow \mathbb{C}^{N^{\prime}}$, where $U \subset \mathbb{C}^{N}$ is an open set, be a holomorphic map $\left(N, N^{\prime} \in \mathbb{N}:=\{1,2,3, \ldots\}\right)$. Assume that a compact set 
$\emptyset \neq K \subset \mathbb{C}^{N}$ has the HCP property and $\hat{K} \subset U$. Under what conditions does it happen that $h(K)$ has the HCP property?

Recall that $\hat{K}$ denotes the polynomially convex hull of $K$ :

$$
\hat{K}:=\left\{z \in \mathbb{C}^{N}:|Q(z)| \leq\|Q\|_{K} \text { for each } Q \in \mathbb{C}\left[z_{1}, \ldots, z_{N}\right]\right\} .
$$

(We set $\hat{\emptyset}:=\emptyset$.) If $\hat{K}=K$, then we say that $K$ is polynomially convex. Occasionally, we will write $K$ instead of $\hat{K}$. It is well known that:

- A compact set $K \subset \mathbb{C}$ is polynomially convex if and only if $\mathbb{C} \backslash K$ is connected; see [22, Corollary 1.3.2].

- Each compact subset of $\mathbb{R}^{N}$ is polynomially convex in $\mathbb{C}^{N}$; see [23, Lemma 5.4.1].

Remark 1.6 Note that, in Problem 1.5, the assumption that $\hat{K} \subset U$ is quite natural. Indeed, consider the simplest example: take $a \in \mathbb{C}$ with $|a|>1$, and put

$$
U:=\mathbb{C} \backslash\{0\}, \quad h: U \ni z \mapsto \frac{1}{z} \in \mathbb{C}, \quad K:=\{|z|=1\} \cup\left\{\frac{1}{a}\right\} \subset \mathbb{C}
$$

Moreover, for each $n \in \mathbb{N}$, set

$$
Q_{n}(z):=z^{n}(z-a), \quad a_{n}:=a+\frac{1}{n}
$$

Since $\hat{K}=\{|z| \leq 1\}$, it follows that

$$
\Phi_{K}(z)=\Phi_{\hat{K}}(z)=\max \{1,|z|\}
$$

for all $z \in \mathbb{C}$; see Example 1.3. In particular, the set $K$ has the HCP property. On the other hand,

$$
\liminf _{n \rightarrow+\infty} \Phi_{h(K)}\left(a_{n}\right) \geq \lim _{n \rightarrow+\infty} \sqrt[n+1]{\frac{\left|Q_{n}\left(a_{n}\right)\right|}{|a|+1}}=|a|>1=\Phi_{h(K)}(a),
$$

and hence $\Phi_{h(K)}$ (and $\left.V_{h(K)}\right)$ is not even continuous.

Problem 1.5 is well known to specialists in the field and, at least since the 1980s, a number of attempts have been made to give a solution. We should mention here a result due to Pleśniak [37], which reads as follows. Let $h: U \rightarrow \mathbb{C}^{N}$, where $U \subset \mathbb{C}^{N}$ $i$ an open set, be a holomorphic map $(N \in \mathbb{N})$. Assume that a compact, polynomially convex set $\emptyset \neq K \subset U$ has the HCP property and $h$ is nonsingular (see Definition $1.7)$ on $K$. Then $h(K)$ has the HCP property as well. To my knowledge, except for this result of Pleśniak, which goes back to 1988, there has been no satisfactory progress on Problem 1.5. In this paper, we prove Theorem 1.8, which gives a complete solution of this problem. Before we state it, however, we set up terminology. 
Definition 1.7 Let $h: U \rightarrow \mathbb{C}^{N^{\prime}}$, where $U \subset \mathbb{C}^{N}$ is an open set, be a holomorphic $\operatorname{map}\left(N, N^{\prime} \in \mathbb{N}\right)$.

- We say that $h$ is nondegenerate if, for each connected component $U_{l}$ of $U$, there exists $\zeta_{\iota} \in U_{l}$ such that rank $d_{\zeta_{l}} h=N^{\prime}$.

- Let $K \subset U$. We say that $h$ is nonsingular on $K$ if $N=N^{\prime}$ and, for each $\zeta \in K$, we have rank $d_{\zeta} h=N$.

Theorem 1.8 Let $h: U \rightarrow \mathbb{C}^{N^{\prime}}$, where $U \subset \mathbb{C}^{N}$ is an open set, be a holomorphic $\operatorname{map}\left(N, N^{\prime} \in \mathbb{N}\right)$. Set

$$
I_{*}:=\left\{\iota \in I:\left.h\right|_{U_{\iota}} \text { is nondegenerate }\right\}, \quad U_{*}:=\bigcup_{\iota \in I_{*}} U_{\iota},
$$

where $\left\{U_{l}\right\}_{l \in I}$ is the family of all connected components of $U$. Assume that a compact set $\emptyset \neq K \subset \mathbb{C}^{N}$ has the HCP property and $\hat{K} \subset U$. Then the following three statements are equivalent:

(i) $h(K)$ has the HCP property;

(ii) $h(K)$ is L-regular (that is, $V_{h(K)}$ is continuous);

(iii) $h(K) \subset h\left(K \cap U_{*}\right)$.

In particular, condition (iii) is the answer to Problem 1.5. Obviously, this condition is automatically satisfied if $h$ nondegenerate. Hence, we get the following result.

Theorem 1.9 Let $h: U \rightarrow \mathbb{C}^{N^{\prime}}$, where $U \subset \mathbb{C}^{N}$ is an open set, be a nondegenerate holomorphic map $\left(N, N^{\prime} \in \mathbb{N}\right)$. Assume that a compact set $\emptyset \neq K \subset \mathbb{C}^{N}$ has the $H C P$ property and $\hat{K} \subset U$. Then $h(K)$ has the HCP property as well.

One of the most important applications of the HCP property concerns multivariate polynomial inequalities. More precisely, the HCP property is a sufficient condition for Markov's inequality.

Definition 1.10 We say that a compact set $\emptyset \neq K \subset \mathbb{C}^{N}$ satisfies Markov's inequality (or: is a Markov set) if there exist $\varepsilon, C>0$ such that, for each polynomial $Q \in$ $\mathbb{C}\left[z_{1}, \ldots, z_{N}\right]$ and each $\alpha=\left(\alpha_{1}, \ldots, \alpha_{N}\right) \in \mathbb{N}_{0}^{N}$,

$$
\left\|D^{\alpha} Q\right\|_{K} \leq\left(C(\operatorname{deg} Q)^{\varepsilon}\right)^{|\alpha|}\|Q\|_{K},
$$

where $\mathbb{N}_{0}:=\mathbb{N} \cup\{0\}, D^{\alpha} Q:=\frac{\partial^{|\alpha|} Q}{\partial z_{1}^{\alpha_{1}} \ldots \partial z_{N}^{\alpha_{N}}}$ and $|\alpha|:=\alpha_{1}+\cdots+\alpha_{N}$.

This is a generalization of the classical inequality due to Markov: If $Q$ is a polynomial of one variable, then

$$
\left\|Q^{\prime}\right\|_{[-1,1]} \leq(\operatorname{deg} Q)^{2}\|Q\|_{[-1,1]}
$$

It is perhaps worth remarking that, for a compact set $\emptyset \neq K \subset \mathbb{C}^{N}$, Markov's inequality (Definition 1.10) is equivalent to the following condition: there exist 
$\varepsilon, D, M>0$ such that, for each polynomial $Q \in \mathbb{C}\left[z_{1}, \ldots, z_{N}\right]$ with $\operatorname{deg} Q \leq n$ $(n \in \mathbb{N})$,

$$
\|Q\|_{\left.K_{(D n}-\varepsilon\right)} \leq M\|Q\|_{K}
$$

This follows easily from Cauchy's inequalities and Taylor's formula.

Markov type inequalities and related topics have been studied by many authors; see for instance [1-3,6-15,19-21,24,29,30,32,34,35,38,40,47]. In this paper, we are interested in the following problem.

Problem 1.11 Let $h: U \rightarrow \mathbb{C}^{N^{\prime}}$, where $U \subset \mathbb{C}^{N}$ is an open set, be a nondegenerate holomorphic map $\left(N, N^{\prime} \in \mathbb{N}\right)$. Assume that a compact set $\emptyset \neq K \subset \mathbb{C}^{N}$ satisfies Markov's inequality and $\hat{K} \subset U$. Under what conditions does it happen that $h(K)$ satisfies Markov's inequality?

This problem has attracted considerable interest over the past decades, and certain partial results have been produced:

- Baran and Pleśniak [3, Theorem 2.5]. If additionally $N=N^{\prime}, K$ is polynomially convex, $h: U \rightarrow \mathbb{C}^{N}$ is nonsingular on $K$, and $h(K)$ is nonpluripolar, then $h(K)$ satisfies Markov's inequality.

- Baran, Białas-Cież and Milówka [2, Theorem 4.2]. If additionally $N=N^{\prime}=1$ and $K$ is polynomially convex, then $h(K)$ satisfies Markov's inequality.

- Pierzchała [35, Theorem 1.4]. If additionally $U=\mathbb{C}^{N}$ and $h$ is a polynomial map, then $h(K)$ satisfies Markov's inequality. However, the proof of this result essentially relies on the assumption that $h$ is a polynomial map and cannot be adapted to holomorphic maps.

In the present article, we give the following answer to the question raised in Problem 1.11 .

Theorem 1.12 Let $h: U \rightarrow \mathbb{C}^{N^{\prime}}$, where $U \subset \mathbb{C}^{N}$ is an open set, be a nondegenerate holomorphic map $\left(N, N^{\prime} \in \mathbb{N}\right)$. Assume that a compact set $K \subset \mathbb{C}^{N}$ satisfies Markov's inequality, $\hat{K} \subset U$, and $h(K)$ is a nonpluripolar subset of $\mathbb{C}^{N^{\prime}}$. Then $h(K)$ satisfies Markov's inequality as well.

Theorem 1.12 "almost" solves Problem 1.11. The only issue here is the nonpluripolarity assumption on the set $h(K)$. However, this assumption is really weak (in particular, pluripolar sets have Lebesgue measure zero; see [23, Corollary 2.9.10]). Furthermore, it is conjectured that all (nonempty) Markov sets are nonpluripolar. If this conjecture is true, then the set $K$ of Theorem 1.12 is nonpluripolar and an elementary argument shows (cf. the proof of [36, Lemma 2.5]) that $h(K)$ is nonpluripolar as well.

The proofs of Theorems 1.8 and 1.12 involve several ingredients. One of them is common to both proofs. It is the following result describing the geometry of nondegenerate holomorphic mappings.

Theorem 1.13 Let $h: U \rightarrow \mathbb{C}^{N^{\prime}}$, where $U \subset \mathbb{C}^{N}$ is an open set, be a nondegenerate holomorphic map $\left(N, N^{\prime} \in \mathbb{N}\right)$. Assume that $K \subset U$ is a compact set. Then there 
exist $\varkappa, \theta, t_{*}>0$ and $q \in \mathbb{N}$ such that, for each $a \in K, \mathbb{D}\left(a, t_{*}\right) \subset U$ and we can choose a polynomial map $Q_{a}: \mathbb{C} \rightarrow \mathbb{C}^{N^{\prime}}$ with $\operatorname{deg} Q_{a} \leq q$ such that

- $Q_{a}(0)=h(a)$,

- $\operatorname{dist}\left(Q_{a}(t), \mathbb{C}^{N^{\prime}} \backslash h(\mathbb{D}(a, t))\right) \geq \theta t^{\varkappa}$ for all $t \in\left(0, t_{*}\right]$.

Recall that in our notation $\mathbb{D}\left(a, t_{*}\right):=\left\{z \in \mathbb{C}^{N}:|z-a| \leq t_{*}\right\}$.

\section{Proof of Theorem 1.13}

\subsection{Notation}

For any $N, N^{\prime} \in \mathbb{N}$, put

$$
\mathcal{A}\left(N, N^{\prime}\right):=\left\{\sigma:\left\{1, \ldots, N^{\prime}\right\} \rightarrow\{1, \ldots, N\}: 1 \leq \sigma(1)<\cdots<\sigma\left(N^{\prime}\right) \leq N\right\} .
$$

If $N^{\prime}<N$ and $\sigma \in \mathcal{A}\left(N, N^{\prime}\right)$, then $\bar{\sigma}$ denotes the unique element of $\mathcal{A}\left(N, N-N^{\prime}\right)$ such that

$$
\left\{\sigma(1), \ldots, \sigma\left(N^{\prime}\right)\right\} \cup\left\{\bar{\sigma}(1), \ldots, \bar{\sigma}\left(N-N^{\prime}\right)\right\}=\{1, \ldots, N\}
$$

\subsection{Key lemmas}

We have divided the proof into a sequence of lemmas. The last lemma is the desired conclusion.

Take an open and bounded set $\Omega \subset \mathbb{C}^{N}$ such that $K \subset \Omega, \bar{\Omega} \subset U$ and $\Omega$ is a semianalytic subset of $\mathbb{R}^{2 N}$. Moreover, set

$$
E:=\left\{\zeta \in \Omega: \operatorname{rank} d_{\zeta} h=N^{\prime}\right\} .
$$

Lemma 2.1 There exist $\theta_{1}, \theta_{2}, v>0$ and $d \in \mathbb{N}$ such that, for each $a \in \bar{E}$, we can choose a polynomial map $R_{a}: \mathbb{C} \rightarrow \mathbb{C}^{N}$ with $\operatorname{deg} R_{a} \leq d$, satisfying the following conditions:

(S1) $\operatorname{dist}\left(R_{a}(t), \mathbb{C}^{N} \backslash E\right) \geq \theta_{1} t^{v}$ for all $t \in[0,1]$,

(S2) $\left|R_{a}(t)-a\right| \leq \theta_{2} t$ for all $t \in[0,1]$. In particular, $R_{a}(0)=a$.

Lemma 2.2 There exist $\theta_{3}, \theta_{4}, \omega>0$ such that, for each $a \in \bar{E}$ and each $t \in[0,1]$,

$$
\theta_{3} \geq \sum_{\sigma \in \mathcal{A}\left(N, N^{\prime}\right)}\left|\frac{\partial\left(h_{1}, \ldots, h_{N^{\prime}}\right)}{\partial\left(z_{\sigma(1)}, \ldots, z_{\sigma\left(N^{\prime}\right)}\right)}\left(R_{a}(t)\right)\right| \geq \theta_{4} t^{\omega}
$$

Lemma 2.3 There exist $\theta_{5}, \theta_{6}, \varkappa>0$ such that, for each $a \in \bar{E}$ and each $t \in(0,1]$, $\mathbb{D}\left(R_{a}(t), \theta_{5}\right) \subset U$ and

$$
\mathbb{D}\left(h\left(R_{a}(t)\right), \theta_{6} t^{\varkappa}\right) \subset h\left(\mathbb{D}\left(R_{a}(t), \theta_{5} t\right)\right) .
$$


Lemma 2.4 There exist $\theta, t_{*}>0$ and $q \in \mathbb{N}$ such that, for each $a \in K, \mathbb{D}\left(a, t_{*}\right) \subset U$ and we can choose a polynomial map $Q_{a}: \mathbb{C} \rightarrow \mathbb{C}^{N^{\prime}}$ with $\operatorname{deg} Q_{a} \leq q$ such that

- $Q_{a}(0)=h(a)$,

- $\operatorname{dist}\left(Q_{a}(t), \mathbb{C}^{N^{\prime}} \backslash h(\mathbb{D}(a, t))\right) \geq \theta t^{\varkappa}$ for all $t \in\left(0, t_{*}\right]$.

\subsection{Proofs of key lemmas}

Proof of Lemma 2.1 Set

$$
Y:=\left\{\zeta \in U: \operatorname{rank} d_{\zeta} h<N^{\prime}\right\}
$$

Note that

$$
Y=\bigcap_{\sigma \in \mathcal{A}\left(N, N^{\prime}\right)}\left\{\zeta \in U: \frac{\partial\left(h_{1}, \ldots, h_{N^{\prime}}\right)}{\partial\left(z_{\sigma(1)}, \ldots, z_{\sigma\left(N^{\prime}\right)}\right)}(\zeta)=0\right\}
$$

It follows that $Y$ is a closed and semianalytic subset of $U \subset \mathbb{R}^{2 N}$, which implies that $E=\Omega \backslash Y$ is an open, bounded and semianalytic subset of $\mathbb{R}^{2 N}$. By [29, Theorem 6.4], there exist $\theta_{1}, v>0$ and $d \in \mathbb{N}$ such that, for each $x \in \bar{E}$, we can choose a polynomial map $P_{x}: \mathbb{R} \rightarrow \mathbb{R}^{2 N}$ with $\operatorname{deg} P_{x} \leq d$, satisfying the following conditions:

- $P_{x}(0)=x$,

- $\operatorname{dist}\left(P_{x}(t), \mathbb{R}^{2 N} \backslash E\right) \geq \theta_{1} t^{v}$ for all $t \in[0,1]$.

Take $\varphi_{1}, \ldots, \varphi_{d}: \bar{E} \rightarrow \mathbb{R}^{2 N}$ such that, for each $x \in \bar{E}$ and each $t \in \mathbb{R}$,

$$
P_{x}(t)=x+\varphi_{1}(x) t+\cdots+\varphi_{d}(x) t^{d} .
$$

By [30, Lemma 3.1], the maps $\varphi_{1}, \ldots, \varphi_{d}$ are bounded, and hence

$$
C:=\left\|\varphi_{1}\right\|_{\bar{E}}+\cdots+\left\|\varphi_{d}\right\|_{\bar{E}}<+\infty .
$$

For each $a \in \bar{E}$ and $t \in \mathbb{R}$, set $R_{a}(t):=\chi_{N}\left(P_{a}(t)\right)$, where

$$
\chi_{N}: \mathbb{R}^{2 N} \ni\left(u_{1}, v_{1}, \ldots, u_{N}, v_{N}\right) \mapsto\left(u_{1}+i v_{1}, \ldots, u_{N}+i v_{N}\right) \in \mathbb{C}^{N} .
$$

It is straightforward to see that conditions (S1) and (S2) hold with $\theta_{2}:=\sqrt{2} C$.

Proof of Lemma 2.2 Note that, for each $a \in \bar{E}$ and each $t \in[0,1], R_{a}(t) \in \bar{E}$, and hence the first required estimate holds with the constant

$$
\theta_{3}:=\max _{\zeta \in \bar{E}}\left(\sum_{\sigma \in \mathcal{A}\left(N, N^{\prime}\right)}\left|\frac{\partial\left(h_{1}, \ldots, h_{N^{\prime}}\right)}{\partial\left(z_{\sigma(1)}, \ldots, z_{\sigma\left(N^{\prime}\right)}\right)}(\zeta)\right|\right) .
$$

Let $Y$ be as in the proof of Lemma 2.1. There are two cases to consider. 
CASE 1: $Y=\emptyset$. Then the second required estimate holds with $\omega:=1$ and

$$
\theta_{4}:=\min _{\zeta \in \bar{E}}\left(\sum_{\sigma \in \mathcal{A}\left(N, N^{\prime}\right)}\left|\frac{\partial\left(h_{1}, \ldots, h_{N^{\prime}}\right)}{\partial\left(z_{\sigma(1)}, \ldots, z_{\sigma\left(N^{\prime}\right)}\right)}(\zeta)\right|\right)
$$

CASE 2: $Y \neq \varnothing$. By [28, p. 243], there exist $C_{1}, \omega_{1}>0$ such that, for each $\zeta \in \bar{E}$,

$$
\sum_{\sigma \in \mathcal{A}\left(N, N^{\prime}\right)}\left|\frac{\partial\left(h_{1}, \ldots, h_{N^{\prime}}\right)}{\partial\left(z_{\sigma(1)}, \ldots, z_{\sigma\left(N^{\prime}\right)}\right)}(\zeta)\right| \geq C_{1} \operatorname{dist}(\zeta, Y)^{\omega_{1}}
$$

Set $\theta_{4}:=C_{1} \theta_{1}^{\omega_{1}}$ and $\omega:=v \omega_{1}$, where $\theta_{1}, v>0$ are of Lemma 2.1. Fix $a \in \bar{E}$ and $t \in[0,1]$. By (2.1) and Lemma 2.1 (condition (S1)), we get

$$
\begin{aligned}
\sum_{\sigma \in \mathcal{A}\left(N, N^{\prime}\right)}\left|\frac{\partial\left(h_{1}, \ldots, h_{N^{\prime}}\right)}{\partial\left(z_{\sigma(1)}, \ldots, z_{\sigma\left(N^{\prime}\right)}\right)}\left(R_{a}(t)\right)\right| & \geq C_{1}\left(\operatorname{dist}\left(R_{a}(t), Y\right)\right)^{\omega_{1}} \\
& \geq C_{1}\left(\operatorname{dist}\left(R_{a}(t), \mathbb{C}^{N} \backslash E\right)\right)^{\omega_{1}} \geq \theta_{4} t^{\omega},
\end{aligned}
$$

which yields the second required estimate.

Proof of Lemma 2.3 Let $\theta_{3}, \theta_{4}, \omega>0$ be of Lemma 2.2. Take $\epsilon \in(0,1)$, and also take $r_{0}>0$ such that $E_{\left(r_{0}\right)} \subset U$. Set

$$
\begin{aligned}
& \eta_{1}:=\max _{1 \leq j \leq N^{\prime}}\left(\sum_{1 \leq k \leq N}\left\|\frac{\partial h_{j}}{\partial z_{k}}\right\|_{E}\right), \\
& \eta_{2}:=\max _{1 \leq j \leq N^{\prime}}\left(\sum_{\substack{1 \leq k \leq N \\
1 \leq \nu \leq N}}\left\|\frac{\partial^{2} h_{j}}{\partial z_{k} \partial z_{v}}\right\|_{E_{\left(r_{0}\right)}}\right) .
\end{aligned}
$$

Furthermore, fix $a \in \bar{E}, t \in(0,1]$, and put $b=b(a, t):=R_{a}(t)$. Note that $b \in E$. By Lemma 2.2, there exists $\sigma \in \mathcal{A}\left(N, N^{\prime}\right)$ such that

$$
\theta_{3} \geq\left|\frac{\partial\left(h_{1}, \ldots, h_{N^{\prime}}\right)}{\partial\left(z_{\sigma(1)}, \ldots, z_{\sigma\left(N^{\prime}\right)}\right)}(b)\right| \geq \frac{\theta_{4} t^{\omega}}{\left(\begin{array}{c}
N \\
N^{\prime}
\end{array}\right)}
$$

Define $H_{\sigma}: U \rightarrow \mathbb{C}^{N}$ by the formula

$$
H_{\sigma}(z):= \begin{cases}\left(h(z), z_{\bar{\sigma}(1)}, \ldots, z_{\bar{\sigma}\left(N-N^{\prime}\right)}\right) & \text { if } N^{\prime}<N \\ h(z) & \text { if } N^{\prime}=N\end{cases}
$$


Note that $d_{b} H_{\sigma}: \mathbb{C}^{N} \rightarrow \mathbb{C}^{N}$ is an isomorphism, because

$$
\left|\operatorname{Jac} H_{\sigma}(b)\right|=\left|\frac{\partial\left(h_{1}, \ldots, h_{N^{\prime}}\right)}{\partial\left(z_{\sigma(1)}, \ldots, z_{\sigma\left(N^{\prime}\right)}\right)}(b)\right| \neq 0 \text {. }
$$

Set $\varrho_{b}:=1 /\left\|\left(d_{b} H_{\sigma}\right)^{-1}\right\|$. Here, and throughout this proof, \|\| denotes the operator norm.

Note that, for each $\zeta \in E$,

$$
\max _{\substack{1 \leq j \leq N \\ 1 \leq k \leq N}}\left|\frac{\partial H_{\sigma, j}}{\partial z_{k}}(\zeta)\right| \leq \max \left\{\eta_{1}, 1\right\}
$$

where

$$
H_{\sigma, j}:= \begin{cases}h_{j} & \text { if } 1 \leq j \leq N^{\prime} \\ z_{\bar{\sigma}\left(j-N^{\prime}\right)} & \text { if } N^{\prime}+1 \leq j \leq N\end{cases}
$$

For $1 \leq j \leq N, 1 \leq k \leq N$, let $\Delta_{j k}(\zeta) \in \mathbb{C}$ denote the entries of the classical adjoint of the jacobian matrix of the map $H_{\sigma}$ at the point $\zeta$. It follows from (2.3) that, for each $\zeta \in E,\left|\Delta_{j k}(\zeta)\right| \leq M$, where $M=M\left(\eta_{1}, N\right)$ is a positive constant depending only on $\eta_{1}$ and $N$. For example, we can take

$$
M:=\left(\max \left\{\eta_{1}, 1\right\}\right)^{N-1}(N-1)^{(N-1) / 2} .
$$

Thus, we have in particular the following estimate:

$$
\left\|\left(d_{b} H_{\sigma}\right)^{-1}\right\| \leq \frac{M N}{\left|\operatorname{Jac} H_{\sigma}(b)\right|}=\frac{M N}{\left|\frac{\partial\left(h_{1}, \ldots, h_{N^{\prime}}\right)}{\partial\left(z_{\sigma(1)}, \ldots, z_{\sigma\left(N^{\prime}\right)}\right)}(b)\right|},
$$

and hence

$$
\varrho_{b} \geq \frac{\left|\frac{\partial\left(h_{1}, \ldots, h_{N^{\prime}}\right)}{\partial\left(z_{\sigma(1)}, \ldots, z_{\sigma\left(N^{\prime}\right)}\right)}(b)\right|}{M N}
$$

Set

$$
\begin{aligned}
\eta & :=\max \left\{\frac{\epsilon \eta_{1}}{r_{0}}, \frac{\epsilon}{r_{0}}, \eta_{2}, \frac{\epsilon \theta_{3}}{r_{0} M N}\right\}, \\
\theta_{5} & :=\frac{\epsilon \theta_{3}}{M N \eta}, \\
\theta_{6} & :=\frac{\epsilon(1-\epsilon)}{\eta}\left(\frac{\theta_{4}}{\left(\begin{array}{l}
N \\
N^{\prime}
\end{array}\right) M N}\right)^{2},
\end{aligned}
$$




$$
\begin{aligned}
& \varkappa:=2 \omega+1, \\
& r=r(a, t):=\frac{\epsilon t}{M N \eta}\left|\frac{\partial\left(h_{1}, \ldots, h_{N^{\prime}}\right)}{\partial\left(z_{\sigma(1)}, \ldots, z_{\sigma\left(N^{\prime}\right)}\right)}(b)\right| .
\end{aligned}
$$

Note that $\theta_{5}$ and $\theta_{6}$ depend neither on $a$ nor $t$. Moreover, (2.4) gives

$$
r \leq \frac{\epsilon \varrho_{b}}{\eta}
$$

Clearly, $\left\|d_{b} H_{\sigma}\right\| \leq \max \left\{\eta_{1}, 1\right\}$, and hence, by (2.5),

$$
r \leq \frac{\epsilon \varrho_{b}}{\eta} \leq \frac{r_{0} \varrho_{b}}{\max \left\{\eta_{1}, 1\right\}}=\frac{r_{0}}{\max \left\{\eta_{1}, 1\right\}\left\|\left(d_{b} H_{\sigma}\right)^{-1}\right\|} \leq \frac{r_{0}\left\|d_{b} H_{\sigma}\right\|}{\max \left\{\eta_{1}, 1\right\}} \leq r_{0}
$$

Consequently, $r \leq r_{0}$ and $\mathbb{D}(b, r) \subset E_{\left(r_{0}\right)} \subset U$.

Put $g_{b}:=d_{b} \bar{H}_{\sigma}-H_{\sigma}: U \rightarrow \mathbb{C}^{N}$. Observe that, for each $\zeta \in \mathbb{D}(b, r)$,

$$
\left\|d_{\zeta} g_{b}\right\| \leq \eta_{2} r
$$

Indeed, $d_{\zeta} g_{b}=d_{b} H_{\sigma}-d_{\zeta} H_{\sigma}$ and therefore

$$
\left\|d_{\zeta} g_{b}\right\|=\left\|d_{b} H_{\sigma}-d_{\zeta} H_{\sigma}\right\| \leq \eta_{2}|\zeta-b| \leq \eta_{2} r
$$

which gives (2.6).

Consider the map $\psi_{b}:=\left(d_{b} H_{\sigma}\right)^{-1} \circ g_{b}$. For $z, z^{\prime} \in \mathbb{D}(b, r)$, we have

$$
\begin{aligned}
\left|\psi_{b}(z)-\psi_{b}\left(z^{\prime}\right)\right| & \leq\left\|\left(d_{b} H_{\sigma}\right)^{-1}\right\| \cdot\left|g_{b}(z)-g_{b}\left(z^{\prime}\right)\right| \\
& \leq \eta_{2} r\left\|\left(d_{b} H_{\sigma}\right)^{-1}\right\| \cdot\left|z-z^{\prime}\right| \\
& \leq \frac{\eta r}{\varrho_{b}}\left|z-z^{\prime}\right| \leq \epsilon\left|z-z^{\prime}\right|
\end{aligned}
$$

and hence

$$
\left|\psi_{b}(z)-\psi_{b}\left(z^{\prime}\right)\right| \leq \epsilon\left|z-z^{\prime}\right|
$$

[17, Theorem 4.4.1], together with (2.7), yields

$$
\mathbb{D}\left(\left(d_{b} H_{\sigma}\right)^{-1}\left(H_{\sigma}(b)\right),(1-\epsilon) r\right) \subset\left(d_{b} H_{\sigma}\right)^{-1}\left(H_{\sigma}(\mathbb{D}(b, r))\right) .
$$

But, for each $z \in \mathbb{C}^{N}$ and $\tau>0$,

$$
\mathbb{D}\left(d_{b} H_{\sigma}(z), \varrho_{b} \tau\right) \subset d_{b} H_{\sigma}(\mathbb{D}(z, \tau)) .
$$


Combining this with (2.8), we get

$$
\mathbb{D}\left(H_{\sigma}(b), \varrho_{b}(1-\epsilon) r\right) \subset H_{\sigma}(\mathbb{D}(b, r)) .
$$

Let

$$
\pi: \mathbb{C}^{N} \ni\left(u_{1}, \ldots, u_{N}\right) \mapsto\left(u_{1}, \ldots, u_{N^{\prime}}\right) \in \mathbb{C}^{N^{\prime}}
$$

The above inclusion implies that

$$
\pi\left(\mathbb{D}\left(H_{\sigma}(b), \varrho_{b}(1-\epsilon) r\right)\right) \subset\left(\pi \circ H_{\sigma}\right)(\mathbb{D}(b, r)),
$$

and hence

$$
\mathbb{D}\left(h(b), \varrho_{b}(1-\epsilon) r\right) \subset h(\mathbb{D}(b, r))
$$

Since $\varrho_{b}(1-\epsilon) r \geq \theta_{6} t^{\varkappa}$ (see (2.2) and (2.4)) and $r \leq \theta_{5} t$ (see (2.2)), we get

$$
\mathbb{D}\left(h(b), \theta_{6} t^{\varkappa}\right) \subset h\left(\mathbb{D}\left(b, \theta_{5} t\right)\right)
$$

which is the desired conclusion.

Proof of Lemma 2.4 Let $\theta_{2}>0, d \in \mathbb{N}$ be of Lemma 2.1 and let $\theta_{5}, \theta_{6}, \varkappa>0$ be of Lemma 2.3. Take $q_{0} \in \mathbb{N}$ such that $q_{0}>\varkappa-1$ and put

$$
\theta_{7}:=\frac{1}{\theta_{2}+\theta_{5}}, \quad \theta:=\frac{\theta_{6} \theta_{7}^{\varkappa}}{2}, \quad q:=d q_{0} .
$$

For each $u \in(-1,1)$, set

$$
\varphi(u):=\sum_{\nu=q_{0}+1}^{+\infty}\left(\begin{array}{c}
v+N-1 \\
v
\end{array}\right) u^{\nu-q_{0}-1} .
$$

Furthermore, take $r_{0}>0$ such that $E_{\left(r_{0}\right)} \subset U$.

Clearly, there exists $t_{*}>0$ such that $t_{*} \leq \min \left\{r_{0}, 1 / \theta_{7}\right\}$ and

$$
t_{*}^{q_{0}+1-\varkappa} \varphi\left(\frac{\theta_{2} \theta_{7} t_{*}}{r_{0}}\right) \leq \frac{\theta}{\|h\|_{E_{\left(r_{0}\right)}}}\left(\frac{r_{0}}{\theta_{2} \theta_{7}}\right)^{q_{0}+1} .
$$

Note that $t_{*} \leq r_{0}<r_{0} /\left(\theta_{2} \theta_{7}\right)$.

Fix $a \in \bar{E}$ (observe that $K \subset \Omega \subset \bar{E}$ ). Define $Q_{a}=\left(Q_{a, 1}, \ldots, Q_{a, N^{\prime}}\right): \mathbb{C} \rightarrow$ $\mathbb{C}^{N^{\prime}}$ by the formula

$$
Q_{a, j}(t):=\sum_{\substack{\alpha \in \mathbb{N}_{0}^{N} \\|\alpha| \leq q_{0}}} \frac{D^{\alpha} h_{j}(a)}{\alpha !}\left(R_{a}\left(\theta_{7} t\right)-a\right)^{\alpha},
$$


where $R_{a}: \mathbb{C} \rightarrow \mathbb{C}^{N}$ is the polynomial map of Lemma 2.1. Clearly, deg $Q_{a} \leq q$ and $Q_{a}(0)=h(a)$; see Lemma 2.1. Note also that, for each $t \in\left[0, t_{*}\right]$,

$$
\left|h\left(R_{a}\left(\theta_{7} t\right)\right)-Q_{a}(t)\right| \leq \theta t^{\varkappa}
$$

Indeed, the estimate (S2) of Lemma 2.1 gives

$$
\left|R_{a}\left(\theta_{7} t\right)-a\right| \leq \theta_{2} \theta_{7} t<r_{0}
$$

In particular,

$$
R_{a}\left(\theta_{7} t\right) \in \mathbb{D}\left(a, r_{0}\right) \subset E_{\left(r_{0}\right)} \subset U .
$$

Hence, for each $j \leq N^{\prime}$,

$$
h_{j}\left(R_{a}\left(\theta_{7} t\right)\right)=\sum_{\alpha \in \mathbb{N}_{0}^{N}} \frac{D^{\alpha} h_{j}(a)}{\alpha !}\left(R_{a}\left(\theta_{7} t\right)-a\right)^{\alpha}
$$

Finally, for each $j \leq N^{\prime}$,

$$
\begin{aligned}
& \left|h_{j}\left(R_{a}\left(\theta_{7} t\right)\right)-Q_{a, j}(t)\right| \\
\leq & \sum_{\substack{\alpha \in \mathbb{N}_{0}^{N} \\
|\alpha| \geq q_{0}+1}} \frac{\left|D^{\alpha} h_{j}(a)\right|}{\alpha !}\left|R_{a}\left(\theta_{7} t\right)-a\right|^{|\alpha|} \\
\leq & \sum_{\substack{\alpha \in \mathbb{N}_{0}^{N} \\
|\alpha| \geq q_{0}+1}} \frac{\left|D^{\alpha} h_{j}(a)\right|}{\alpha !}\left(\theta_{2} \theta_{7} t\right)^{|\alpha|} \\
\leq & \sum_{\substack{\alpha \in \mathbb{N}_{0}^{N} \\
|\alpha| \geq q_{0}+1}}\left\|h_{j}\right\|_{E_{\left(r_{0}\right)}}\left(\frac{\theta_{2} \theta_{7} t}{r_{0}}\right)^{|\alpha|} \\
= & \sum_{\nu=q_{0}+1}^{+\infty}\left\|h_{j}\right\|_{E_{\left(r_{0}\right)}}\left(\begin{array}{c}
\nu+N-1 \\
v
\end{array}\right)\left(\frac{\theta_{2} \theta_{7} t}{r_{0}}\right)^{v} \\
\leq & \left\|h_{j}\right\|_{E_{\left(r_{0}\right)}}\left(\frac{\theta_{2} \theta_{7}}{r_{0}}\right)^{q_{0}+1} t^{\varkappa} t_{*}^{q_{0}+1-\varkappa}\left(\frac{\theta_{2} \theta_{7} t_{*}}{r_{0}}\right) \\
\leq & \theta t^{\varkappa}
\end{aligned}
$$

which yields (2.10).

It follows from (2.11) that, for each $t \in\left(0, t_{*}\right]$,

$$
\mathbb{D}\left(R_{a}\left(\theta_{7} t\right), \theta_{5} \theta_{7} t\right) \subset \mathbb{D}(a, t) \subset \mathbb{D}\left(a, t_{*}\right) \subset U
$$


Therefore, for each $t \in\left(0, t_{*}\right]$,

$$
\begin{array}{ll}
\operatorname{dist}\left(Q_{a}(t), \mathbb{C}^{N^{\prime}} \backslash h(\mathbb{D}(a, t))\right) & \\
\geq \operatorname{dist}\left(h\left(R_{a}\left(\theta_{7} t\right)\right), \mathbb{C}^{N^{\prime}} \backslash h(\mathbb{D}(a, t))\right)-\theta t^{\varkappa} & \text { (by (2.10)) } \\
\geq \operatorname{dist}\left(h\left(R_{a}\left(\theta_{7} t\right)\right), \mathbb{C}^{N^{\prime}} \backslash h\left(\mathbb{D}\left(R_{a}\left(\theta_{7} t\right), \theta_{5} \theta_{7} t\right)\right)\right)-\theta t^{\varkappa} & \text { (by (2.14)) } \\
\geq \theta_{6}\left(\theta_{7} t\right)^{\varkappa}-\theta t^{\varkappa}=\theta t^{\varkappa} & \text { (by Lemma 2.3), }
\end{array}
$$

which completes the proof of the lemma (and hence proves Theorem 1.13).

\section{Proof of Theorem 1.9}

Proof of Theorem 1.9 Take an open, bounded set $\Omega \subset \mathbb{C}^{N}$ such that $\hat{K} \subset \Omega$ and $\bar{\Omega} \subset U$. Furthermore, take a compact and polynomially convex set $E \subset \mathbb{C}^{N}$ such that $K \subset \operatorname{Int} E$ and $E \subset \Omega$; cf. [22, Proof of Lemma 2.7.4]. By the uniform version of the Bernstein-Walsh-Siciak theorem (see [36,41] and see also [33]), there exist $C_{1}>0$ and $\rho \in(0,1)$ with the following property: for each holomorphic and bounded function $f: \Omega \rightarrow \mathbb{C}$ and each $v \in \mathbb{N}$, there exists a polynomial $W_{v} \in \mathbb{C}\left[z_{1}, \ldots, z_{N}\right]$ with $\operatorname{deg} W_{v} \leq v$ and such that

$$
\left\|f-W_{v}\right\|_{E} \leq C_{1}\|f\|_{\Omega} \rho^{v}
$$

By Theorem 1.13, there exist $\varkappa, \theta, t_{*}>0$ and $q \in \mathbb{N}$ such that, for each $a \in K$, $\mathbb{D}\left(a, t_{*}\right) \subset U$ and we can choose a polynomial map $Q_{a}: \mathbb{C} \rightarrow \mathbb{C}^{N^{\prime}}$ with $\operatorname{deg} Q_{a} \leq q$ such that

(i) $Q_{a}(0)=h(a)$,

(ii) $\operatorname{dist}\left(Q_{a}(t), \mathbb{C}^{N^{\prime}} \backslash h(\mathbb{D}(a, t))\right) \geq \theta t^{\varkappa}$ for all $t \in\left(0, t_{*}\right]$.

We can clearly assume that $\varkappa \in \mathbb{N}$ and $K_{\left(t_{*}\right)} \subset E$.

Note that the set $h(K)$ is nonpluripolar; cf. [36, Proof of Lemma 2.5]. Therefore, by (1.1) and (1.2), $\Phi_{h(K)}$ is locally bounded in $\mathbb{C}^{N^{\prime}}$. In particular, there exists $k \in \mathbb{N}$ such that

$$
\rho^{k} \sup _{h(\Omega)} \Phi_{h(K)} \leq 1
$$

Since the set $K$ has the HCP property, there exist $C_{2}, \mu>0$ such that, for each $z \in K_{\left(t_{*}\right)}$,

$$
\Phi_{K}(z) \leq 1+C_{2}(\operatorname{dist}(z, K))^{\mu}
$$

Set

$$
\gamma:=\frac{\mu}{\varkappa(2 \mu+1)},
$$




$$
\begin{aligned}
\tau_{*} & :=t_{*}^{\varkappa(2 \mu+1)}, \\
C_{3} & :=2 \sqrt{\frac{2 N^{\prime}-1}{\theta^{1 / \varkappa}}}\left(\sqrt{\tau_{*}^{2 \gamma} \frac{2 N^{\prime}-1}{\theta^{1 / \varkappa}}}+\sqrt{\tau_{*}^{2 \gamma} \frac{2 N^{\prime}-1}{\theta^{1 / \varkappa}}+1}\right), \\
C_{4} & :=\sup _{\tau \in\left(0, \tau_{*}\right]} \frac{\left(1+\max \left\{C_{2}, C_{3}\right\} \tau^{\gamma}\right)^{\max \{q, \varkappa\}+k}-1}{\tau^{\gamma}} \\
& =\frac{\left(1+\max \left\{C_{2}, C_{3}\right\} \tau_{*}^{\gamma}\right)^{\max \{q, \varkappa\}+k}-1}{\tau_{*}^{\gamma}} .
\end{aligned}
$$

We claim that, for each $y \in h(K)_{\left(\tau_{*}\right)}$,

$$
\Phi_{h(K)}(y) \leq 1+C_{4}(\operatorname{dist}(y, h(K)))^{\gamma}
$$

which then proves the theorem.

To see the claim, fix a polynomial $P \in \mathbb{C}\left[y_{1}, \ldots, y_{N^{\prime}}\right]$ with $\operatorname{deg} P \leq n(n \in \mathbb{N})$, and also fix $y \in h(K)_{\left(\tau_{*}\right)}$. Take $a \in K$ such that $\operatorname{dist}(y, h(K))=|y-h(a)|$ and set $\tau:=|y-h(a)|$. Note that $\tau \leq \tau_{*}$. It suffices to show that

$$
|P(y)| \leq\left(1+C_{4} \tau^{\gamma}\right)^{n}\|P\|_{h(K)} .
$$

We may assume that $\tau>0$, because otherwise (3.5) is trivial. Put $r:=\tau^{\gamma / \mu}$ and let $H_{r}: \mathbb{C}^{N^{\prime}+1} \rightarrow \mathbb{C}^{N^{\prime}}$ be the map

$$
\begin{aligned}
H_{r}\left(s, \chi_{1}, \ldots, \chi_{N^{\prime}}\right)= & Q_{a}\left(\frac{r}{N^{\prime}+1}\left(s+\chi_{1}+\cdots+\chi_{N^{\prime}}\right)\right) \\
& +\theta\left(\frac{r}{N^{\prime}+1}\right)^{\varkappa}\left(\left(s-\chi_{1}\right)^{\varkappa}, \ldots,\left(s-\chi_{N^{\prime}}\right)^{\varkappa}\right) .
\end{aligned}
$$

Clearly, $H_{r}$ is a polynomial map with $\operatorname{deg} H_{r} \leq \max \{q, \varkappa\}$. Using (i) and (ii) we easily verify that

$$
H_{r}\left([0,1]^{N^{\prime}+1}\right) \subset h(\mathbb{D}(a, r))
$$

Let $\zeta(y)=\left(\zeta_{1}(y), \ldots, \zeta_{N^{\prime}}(y)\right) \in \mathbb{C}^{N^{\prime}}$ be such that

$$
\theta\left(\frac{r}{N^{\prime}+1}\right)^{\varkappa}\left(\zeta_{1}^{\varkappa}(y), \ldots, \zeta_{N^{\prime}}^{\varkappa}(y)\right)=y-h(a)
$$

Note that

$$
|\zeta(y)| \leq\left(\frac{\tau}{\theta}\right)^{1 / \varkappa} \frac{N^{\prime}+1}{r}
$$


Set

$$
\begin{aligned}
& s(y):=\frac{\zeta_{1}(y)+\cdots+\zeta_{N^{\prime}}(y)}{N^{\prime}+1}, \\
& \chi_{1}(y):=\frac{\zeta_{1}(y)+\cdots+\zeta_{N^{\prime}}(y)}{N^{\prime}+1}-\zeta_{1}(y), \\
& \vdots \\
& \chi_{N^{\prime}}(y):=\frac{\zeta_{1}(y)+\cdots+\zeta_{N^{\prime}}(y)}{N^{\prime}+1}-\zeta_{N^{\prime}}(y) .
\end{aligned}
$$

By (i) and (3.7),

$$
H_{r}\left(s(y), \chi_{1}(y), \ldots, \chi_{N^{\prime}}(y)\right)=y .
$$

On account of (3.8), we have moreover

$$
\left\{\begin{array}{l}
|s(y)| \leq\left(\frac{\tau}{\theta}\right)^{1 / \varkappa} \frac{N^{\prime}}{r} \\
\left|\chi_{1}(y)\right| \leq\left(\frac{\tau}{\theta}\right)^{1 / \varkappa} \frac{2 N^{\prime}-1}{r} \\
\vdots \\
\left|\chi_{N^{\prime}}(y)\right| \leq\left(\frac{\tau}{\theta}\right)^{1 / \varkappa} \frac{2 N^{\prime}-1}{r}
\end{array}\right.
$$

Recall that, for each $w \in \mathbb{C}$,

$$
\Phi_{[-1,1]}(w)=\left|w+\sqrt{w^{2}-1}\right|,
$$

where the square root is so chosen that $\left|w+\sqrt{w^{2}-1}\right| \geq 1$; see the last formula in Example 1.3. Consequently,

$$
\Phi_{[0,1]}(w)=\left|2 w-1+2 \sqrt{w^{2}-w}\right|
$$

and hence

$$
\Phi_{[0,1]}(w) \leq 1+2 \sqrt{|w|}(\sqrt{|w|}+\sqrt{|w|+1})
$$

for all $w \in \mathbb{C}$.

By [44, Proposition 5.9],

$$
\begin{aligned}
& \Phi_{[0,1]^{N^{\prime}+1}}\left(s(y), \chi_{1}(y), \ldots, \chi_{N^{\prime}}(y)\right) \\
& \quad=\max \left\{\Phi_{[0,1]}(s(y)), \Phi_{[0,1]}\left(\chi_{1}(y)\right), \ldots, \Phi_{[0,1]}\left(\chi_{N^{\prime}}(y)\right)\right\}
\end{aligned}
$$




$$
\begin{aligned}
& \leq 1+2 \sqrt{\left(\frac{\tau}{\theta}\right)^{1 / \varkappa} \frac{2 N^{\prime}-1}{r}}\left(\sqrt{\left(\frac{\tau}{\theta}\right)^{1 / \varkappa} \frac{2 N^{\prime}-1}{r}}+\sqrt{\left(\frac{\tau}{\theta}\right)^{1 / \varkappa \frac{2 N^{\prime}-1}{r}+1}}\right) \\
& \leq 1+C_{3} \tau^{\gamma} .
\end{aligned}
$$

Hence

$$
\Phi_{[0,1]^{N^{\prime}+1}}\left(s(y), \chi_{1}(y), \ldots, \chi_{N^{\prime}}(y)\right) \leq 1+C_{3} \tau^{\gamma} .
$$

Note that

$$
|P(y)| \leq\left(1+C_{3} \tau^{\gamma}\right)^{n \max \{q, \varkappa\}}\|P\|_{h(\mathbb{D}(a, r))} .
$$

Indeed,

$$
\begin{aligned}
|P(y)| & =\left|\left(P \circ H_{r}\right)\left(s(y), \chi_{1}(y), \ldots, \chi_{N^{\prime}}(y)\right)\right| \\
& \leq\left(\Phi_{[0,1]^{N^{\prime}+1}}\left(s(y), \chi_{1}(y), \ldots, \chi_{N^{\prime}}(y)\right)\right)^{n \max \{q, \varkappa\}}\left\|P \circ H_{r}\right\|_{[0,1]^{N^{\prime}+1}} \\
& \leq\left(1+C_{3} \tau^{\gamma}\right)^{n \max \{q, \varkappa\}}\left\|P \circ H_{r}\right\|_{[0,1]^{N^{\prime}+1}} \\
& \leq\left(1+C_{3} \tau^{\gamma}\right)^{n \max \{q, \varkappa\}}\|P\|_{h(\mathbb{D}(a, r))}
\end{aligned}
$$

which yields (3.13).

By (3.1), for each $j \in \mathbb{N}$, there exists a polynomial $R_{j} \in \mathbb{C}\left[z_{1}, \ldots, z_{N}\right]$ with $\operatorname{deg} R_{j} \leq j k n$ such that

$$
\left\|P^{j} \circ h-R_{j}\right\|_{E} \leq C_{1}\left\|P^{j} \circ h\right\|_{\Omega} \rho^{j k n} .
$$

Then

$$
\begin{aligned}
\left\|P^{j} \circ h-R_{j}\right\|_{E} & \leq C_{1}\left\|P^{j} \circ h\right\|_{\Omega} \rho^{j k n} \\
& \leq C_{1}\left(\sup _{h(\Omega)} \Phi_{h(K)}\right)^{j n}\|P\|_{h(K)}^{j} \rho^{j k n} \\
& \leq C_{1}\|P\|_{h(K)}^{j}
\end{aligned}
$$

Consequently,

$$
\left\|P^{j} \circ h-R_{j}\right\|_{E} \leq C_{1}\|P\|_{h(K)}^{j} .
$$

Since $r \leq t_{*}$, it follows that $\mathbb{D}(a, r) \subset K_{\left(t_{*}\right)} \subset E$. Therefore,

$$
\begin{aligned}
\|P\|_{h(\mathbb{D}(a, r))}^{j} & \leq\left\|P^{j} \circ h-R_{j}\right\|_{\mathbb{D}(a, r)}+\left\|R_{j}\right\|_{\mathbb{D}(a, r)} \\
& \leq C_{1}\|P\|_{h(K)}^{j}+\left\|R_{j}\right\|_{\mathbb{D}(a, r)}
\end{aligned}
$$




$$
\begin{aligned}
& \leq C_{1}\|P\|_{h(K)}^{j}+\left(\sup _{\mathbb{D}(a, r)} \Phi_{K}\right)^{j k n}\left\|R_{j}\right\|_{K} \\
& \leq C_{1}\|P\|_{h(K)}^{j}+\left(1+C_{2} r^{\mu}\right)^{j k n}\left\|R_{j}\right\|_{K}
\end{aligned}
$$

But

$$
\begin{aligned}
\left\|R_{j}\right\|_{K} & \leq\left\|P^{j} \circ h-R_{j}\right\|_{K}+\left\|P^{j} \circ h\right\|_{K} \\
& \leq\left(C_{1}+1\right)\|P\|_{h(K)}^{j}
\end{aligned}
$$

Therefore,

$$
\|P\|_{h(\mathbb{D}(a, r))}^{j} \leq\left(2 C_{1}+1\right)\left(1+C_{2} r^{\mu}\right)^{j k n}\|P\|_{h(K)}^{j} .
$$

Since $j \in \mathbb{N}$ was arbitrary, we get

$$
\|P\|_{h(\mathbb{D}(a, r))} \leq\left(1+C_{2} r^{\mu}\right)^{k n}\|P\|_{h(K)} .
$$

Together with (3.13), this implies that

$$
\begin{aligned}
|P(y)| & \leq\left(1+C_{3} \tau^{\gamma}\right)^{n \max \{q, \varkappa\}}\|P\|_{h(\mathbb{D}(a, r))} \\
& \leq\left(1+C_{2} r^{\mu}\right)^{k n}\left(1+C_{3} \tau^{\gamma}\right)^{n \max \{q, \varkappa\}}\|P\|_{h(K)} \\
& =\left(1+C_{2} \tau^{\gamma}\right)^{k n}\left(1+C_{3} \tau^{\gamma}\right)^{n \max \{q, \varkappa\}}\|P\|_{h(K)} \\
& \leq\left(1+\max \left\{C_{2}, C_{3}\right\} \tau^{\gamma}\right)^{n(\max \{q, \varkappa\}+k)}\|P\|_{h(K)} \\
& \leq\left(1+C_{4} \tau^{\gamma}\right)^{n}\|P\|_{h(K)} .
\end{aligned}
$$

The above estimates yield (3.5) and hence (3.4). The proof of the theorem is complete, because (3.4) means that $h(K)$ has the HCP property, which is our assertion.

\section{Proof of Theorem 1.8}

Proof of Theorem 1.8 For each $\zeta \in U \backslash U_{*}=\bigcup_{l \in I \backslash I_{*}} U_{l}$, we have rank $d_{\zeta} h \leq N^{\prime}-1$. It follows from [28, p. 254] that $h\left(U \backslash U_{*}\right)$ is a countable union of submanifolds of dimension $\leq N^{\prime}-1$. In particular, the set $h\left(U \backslash U_{*}\right)$ is pluripolar (recall that countable unions of pluripolar sets are pluripolar; see [23, Corollary 4.7.7]) and hence $h\left(K \backslash U_{*}\right)$ is pluripolar as well.

(i) $\Longrightarrow$ (ii). Trivial (see Introduction).

(ii) $\Longrightarrow$ (iii). Assume that $h(K)$ is $L$-regular. To obtain a contradiction, suppose that $h(K) \not \subset h\left(K \cap U_{*}\right)$, and take $b \in h(K) \backslash h\left(K \cap U_{*}\right)$. Note that $h\left(K \cap U_{*}\right) \neq \emptyset$ (otherwise, $h(K)$ would be pluripolar, in contradiction with $V_{h(K)}$ being continuous; see (1.1)) and that $V_{h\left(K \cap U_{*}\right)}(b)>0$ (see [44, Corollary 4.14]). Since $h\left(K \backslash U_{*}\right)$ is 
pluripolar, [23, Theorem 5.2.4 and Corollary 5.2.5] imply that

$$
V_{h(K)}^{*}=V_{h\left(K \backslash U_{*}\right) \cup h\left(K \cap U_{*}\right)}^{*}=V_{h\left(K \cap U_{*}\right)}^{*},
$$

and hence

$$
0=V_{h(K)}(b)=V_{h(K)}^{*}(b)=V_{h\left(K \cap U_{*}\right)}^{*}(b) \geq V_{h\left(K \cap U_{*}\right)}(b)>0,
$$

a contradiction. Therefore, $h(K) \subset h\left(K \cap U_{*}\right)$, which is our claim.

(iii) $\Longrightarrow$ (i). Suppose that (iii) holds, that is, $h(K) \subset h(E)$, where $E:=K \cap U_{*}$. We can, clearly, assume that $U_{*} \neq U$, because otherwise Theorem 1.9 immediately completes the proof. In brief outline, the idea of the proof is the following:

- we use the hypothesis that $K$ has the HCP property (4.1) to show that $E$ satisfies Markov's inequality (4.8);

- then we show, with the aid of (4.1) and (4.8), that $E$ has the HCP property (4.9);

- finally, we apply Theorem 1.9 to the set $E$ and the map $\left.h\right|_{U_{*}}$ to deduce that $h(E)$ has the HCP property (4.11);

- the proof of (i) concludes by observing, via the hypothesis (iii), that $V_{h(K)}=V_{h(E)}$.

The HCP property of $K$ means that there exist $\theta_{1}, \mu>0$ such that, for each $z \in K_{(1)}$,

$$
\Phi_{K}(z) \leq 1+\theta_{1}(\operatorname{dist}(z, K))^{\mu} .
$$

By [46, Remark 3.7], $\mu \leq 1$.

Consider $f: U \rightarrow \mathbb{C}$ given by

$$
f(z):= \begin{cases}1 & \text { if } z \in U_{*} \\ 0 & \text { if } z \in U \backslash U_{*} .\end{cases}
$$

Also, take a compact and polynomially convex set $\Delta \subset \mathbb{C}^{N}$ such that $\hat{K} \subset$ Int $\Delta$ and $\Delta \subset U$; cf. [22, Proof of Lemma 2.7.4]. By [44, Theorem 8.5(1)], there exist $\theta_{2}>0, \rho \in(0,1)$, and a sequence of polynomials $W_{v} \in \mathbb{C}\left[z_{1}, \ldots, z_{N}\right](v \in \mathbb{N})$ with $\operatorname{deg} W_{v} \leq v$ such that, for each $v \in \mathbb{N}$,

$$
\left\|f-W_{v}\right\|_{\Delta} \leq \theta_{2} \rho^{v} .
$$

We can, clearly, assume that $\theta_{2} \geq 1$.

Take $\nu_{0} \in \mathbb{N}$ such that $2 \theta_{2} \rho^{\nu_{0}}<1$. We now show that

$$
\hat{E} \subset U_{*}
$$

To this end, take $c \in \hat{E}$, and suppose, towards a contradiction, that $c \notin U_{*}$. Since $c \in \hat{K} \backslash U_{*} \subset U \backslash U_{*}$, we have from (4.2)

$$
\left\|1-W_{\nu_{0}}\right\|_{E}<\frac{1}{2} \text { and }\left|W_{\nu_{0}}(c)\right|<\frac{1}{2} .
$$


Hence,

$$
\left\|1-W_{\nu_{0}}\right\|_{E}<\left|1-W_{\nu_{0}}(c)\right|,
$$

which implies that $c \notin \hat{E}$, a contradiction.

Similarly, we show that $E$ is nonpluripolar. Indeed, striving for a contradiction, assume that $E$ is pluripolar. Note that $\emptyset \neq h(K) \subset h(E)$, which implies that $E \neq \emptyset$. Take $\tilde{c} \in E$. Then, from (4.2),

$$
\left\|W_{\nu_{0}}\right\|_{K \backslash E}<\frac{1}{2} \text { and }\left|W_{\nu_{0}}(\tilde{c})\right|>\frac{1}{2} .
$$

Hence, $\tilde{c} \notin(K \backslash E)$, and by [23, Theorem 5.2.4 and Corollary 5.2.5],

$$
0=V_{K}(\tilde{c})=V_{K}^{*}(\tilde{c})=V_{E \cup(K \backslash E)}^{*}(\tilde{c})=V_{K \backslash E}^{*}(\tilde{c}) \geq V_{K \backslash E}(\tilde{c})>0,
$$

which is a contradiction.

Nonpluripolarity of $E$ implies that $\Phi_{E}$ is locally bounded in $\mathbb{C}^{N}$; see (1.1) and (1.2). In particular, there exists $m \in \mathbb{N}$ such that $\theta_{2} \rho^{m}<1$ and

$$
\sup _{K} \Phi_{E} \leq \frac{1}{\theta_{2} \rho^{m}}
$$

For each nonconstant polynomial $T \in \mathbb{C}\left[z_{1}, \ldots, z_{N}\right]$ set $P^{T}:=W_{m \operatorname{deg} T} T$. Note that, for each $z \in \Delta \cap U_{*}$,

$$
\left|P^{T}(z)\right| \geq\left(1-\theta_{2} \rho^{m \operatorname{deg} T}\right)|T(z)| \geq\left(1-\theta_{2} \rho^{m}\right)|T(z)| .
$$

Indeed, by (4.2),

$$
\begin{aligned}
\left|P^{T}(z)\right| & =\left|W_{m \operatorname{deg} T}(z)\right| \cdot|T(z)| \geq\left(1-\left|W_{m \operatorname{deg} T}(z)-1\right|\right)|T(z)| \\
& \geq\left(1-\theta_{2} \rho^{m \operatorname{deg} T}\right)|T(z)|
\end{aligned}
$$

We now show that, for each nonconstant polynomial $T \in \mathbb{C}\left[z_{1}, \ldots, z_{N}\right]$,

$$
\left\|P^{T}\right\|_{K} \leq\left(1+\theta_{2} \rho^{m \operatorname{deg} T}\right)\|T\|_{E} \leq\left(1+\theta_{2} \rho^{m}\right)\|T\|_{E} .
$$

To this end, fix $z \in K$. By (4.2),

$$
\begin{aligned}
\left|P^{T}(z)\right| & =\left|W_{m \operatorname{deg} T}(z)\right| \cdot|T(z)| \\
& \leq\left(|f(z)|+\left|f(z)-W_{m \operatorname{deg} T}(z)\right|\right)|T(z)| \\
& \leq\left(|f(z)|+\theta_{2} \rho^{m \operatorname{deg} T}\right)|T(z)| .
\end{aligned}
$$


Thus,

$$
\left|P^{T}(z)\right| \leq \begin{cases}\left(1+\theta_{2} \rho^{m \operatorname{deg} T}\right)\|T\|_{E} & \text { if } z \in E \\ \theta_{2} \rho^{m \operatorname{deg} T}|T(z)| & \text { if } z \in K \backslash E .\end{cases}
$$

Moreover,

$$
\begin{aligned}
\theta_{2} \rho^{m \operatorname{deg} T}|T(z)| & \leq\left(\theta_{2} \rho^{m}\right)^{\operatorname{deg} T}|T(z)| \\
& \leq\left(\theta_{2} \rho^{m} \sup _{K} \Phi_{E}\right)^{\operatorname{deg} T}\|T\|_{E} \\
& \leq\|T\|_{E}
\end{aligned}
$$

Combining the above estimates with (4.7) yields (4.6).

Take $\theta_{3} \in(0,1]$ such that $E_{\left(\theta_{3}\right)} \subset \Delta \cap U_{*}$, and set

$$
\begin{aligned}
\theta_{4} & :=\frac{\exp \left(\theta_{1} \theta_{3}^{\mu}(m+1)\right)}{\theta_{3}} \cdot \frac{1+\theta_{2} \rho^{m}}{1-\theta_{2} \rho^{m}}, \\
\theta_{5} & :=\sup _{t \in\left(0, N \theta_{3} \theta_{4}\right]} \frac{\exp (t)-1}{t}=\frac{\exp \left(N \theta_{3} \theta_{4}\right)-1}{N \theta_{3} \theta_{4}}, \\
\theta_{6} & :=\max \left\{\theta_{1}, \frac{2 \theta_{2} \rho^{m}}{\left(1-\theta_{2} \rho^{m}\right) \theta_{3}^{\mu}}\right\}, \\
\theta_{7} & :=\sup _{t \in\left(0, \theta_{3}^{\mu} \theta_{6}\right]} \frac{(1+t)^{m+2}-1}{t}=\frac{\left(1+\theta_{3}^{\mu} \theta_{6}\right)^{m+2}-1}{\theta_{3}^{\mu} \theta_{6}}, \\
\theta_{8} & :=\max \left\{N \theta_{3}^{1-\mu} \theta_{4} \theta_{5}, \theta_{6} \theta_{7}\right\} .
\end{aligned}
$$

We now show that, for each polynomial $Q \in \mathbb{C}\left[z_{1}, \ldots, z_{N}\right]$ and each $\alpha=$ $\left(\alpha_{1}, \ldots, \alpha_{N}\right) \in \mathbb{N}_{0}^{N}$,

$$
\left\|D^{\alpha} Q\right\|_{E} \leq\left(\theta_{4}(\operatorname{deg} Q)^{1 / \mu}\right)^{|\alpha|}\|Q\|_{E}
$$

(If $Q$ is constant and $\alpha=0 \in \mathbb{N}_{0}^{N}$, then $\left(\theta_{4}(\operatorname{deg} Q)^{1 / \mu}\right)^{|\alpha|}:=1$.) Obviously, it suffices to check (4.8) for $\alpha \in \mathbb{N}_{0}^{N}$ such that $|\alpha|=1$. So, fix such an $\alpha$, and also fix $Q \in \mathbb{C}\left[z_{1}, \ldots, z_{N}\right]$. Put $d:=\operatorname{deg} Q$. We may assume that $d \geq 1$, because (4.8) is trivial for $Q$ being constant.

Then, for each $z \in E$,

$$
\begin{aligned}
\left|D^{\alpha} Q(z)\right| & \leq \frac{d^{1 / \mu}}{\theta_{3}}\|Q\|_{\mathbb{D}\left(z, \theta_{3} d^{-1 / \mu}\right)} \\
& \leq \frac{d^{1 / \mu}}{\theta_{3}\left(1-\theta_{2} \rho^{m}\right)}\left\|P^{Q}\right\|_{\mathbb{D}\left(z, \theta_{3} d^{-1 / \mu}\right)}
\end{aligned}
$$




$$
\begin{aligned}
& \leq \frac{d^{1 / \mu}}{\theta_{3}\left(1-\theta_{2} \rho^{m}\right)}\left(1+\frac{\theta_{1} \theta_{3}^{\mu}}{d}\right)^{d(m+1)}\left\|P^{Q}\right\|_{K} \quad \text { (by (1.3) and (4.1)) } \\
& \leq \frac{d^{1 / \mu} \exp \left(\theta_{1} \theta_{3}^{\mu}(m+1)\right)}{\theta_{3}\left(1-\theta_{2} \rho^{m}\right)}\left\|P^{Q}\right\|_{K} \\
& \leq \frac{d^{1 / \mu} \exp \left(\theta_{1} \theta_{3}^{\mu}(m+1)\right)}{\theta_{3}} \cdot \frac{1+\theta_{2} \rho^{m}}{1-\theta_{2} \rho^{m}}\|Q\|_{E} \quad(\text { by (4.6)) } \\
& =\theta_{4} d^{1 / \mu}\|Q\|_{E}
\end{aligned}
$$

which implies (4.8).

We are now in a position to show that, for each $a \in E_{\left(\theta_{3}\right)}$,

$$
\Phi_{E}(a) \leq 1+\theta_{8}(\operatorname{dist}(a, E))^{\mu} .
$$

To prove this, fix $a \in E_{\left(\theta_{3}\right)}$ and $Q \in \mathbb{C}\left[z_{1}, \ldots, z_{N}\right]$ such that $d:=\operatorname{deg} Q>0$. We need to check that

$$
|Q(a)| \leq\left(1+\theta_{8} \delta^{\mu}\right)^{d}\|Q\|_{E},
$$

where $\delta:=\operatorname{dist}(a, E)$.

CASE $1: \delta \leq \theta_{3} d^{-1 / \mu}$. Take $b \in E$ such that $|a-b|=\delta$. Then

$$
\begin{aligned}
& |Q(a)|=\left|\sum_{\alpha \in \mathbb{N}_{0}^{N}} \frac{D^{\alpha} Q(b)}{\alpha !}(a-b)^{\alpha}\right| \\
& \leq \sum_{\alpha \in \mathbb{N}_{0}^{N}} \frac{\left\|D^{\alpha} Q\right\|_{E}}{\alpha !} \delta^{|\alpha|} \\
& \leq \sum_{\alpha \in \mathbb{N}_{0}^{N}} \frac{\left(\theta_{4} d^{1 / \mu} \delta\right)^{|\alpha|}}{\alpha !}\|Q\|_{E} \\
& =\exp \left(N \theta_{4} d^{1 / \mu} \delta\right)\|Q\|_{E} \\
& =\exp \left(d N \theta_{4}\left(d^{1 / \mu} \delta\right)^{1-\mu} \delta^{\mu}\right)\|Q\|_{E} \\
& \leq \exp \left(d N \theta_{3}^{1-\mu} \theta_{4} \delta^{\mu}\right)\|Q\|_{E} \quad \text { (since } \mu \leq 1 \text { and } \delta \leq \theta_{3} d^{-1 / \mu} \text { ) } \\
& \left.\leq\left(1+N \theta_{3}^{1-\mu} \theta_{4} \theta_{5} \delta^{\mu}\right)^{d}\|Q\|_{E} \quad \text { (since } \delta \leq \theta_{3}\right) \\
& \leq\left(1+\theta_{8} \delta^{\mu}\right)^{d}\|Q\|_{E} \text {, }
\end{aligned}
$$

which yields (4.10) in case 1 .

CASE 2: $\delta>\theta_{3} d^{-1 / \mu}$. Then

$$
|Q(a)| \leq \frac{1}{1-\theta_{2} \rho^{m}}\left|P^{Q}(a)\right|
$$




$$
\begin{aligned}
& \leq \frac{1}{1-\theta_{2} \rho^{m}}\left(\Phi_{K}(a)\right)^{d(m+1)}\left\|P^{Q}\right\|_{K} \\
& \leq \frac{1}{1-\theta_{2} \rho^{m}}\left(1+\theta_{1} \delta^{\mu}\right)^{d(m+1)}\left\|P^{Q^{(m}}\right\|_{K} \\
& \leq \frac{1+\theta_{2} \rho^{m}}{1-\theta_{2} \rho^{m}}\left(1+\theta_{1} \delta^{\mu}\right)^{d(m+1)}\|Q\|_{E} \\
& =\left(1+\frac{2 \theta_{2} \rho^{m}}{1-\theta_{2} \rho^{m}}\right)\left(1+\theta_{1} \delta^{\mu}\right)^{d(m+1)}\|Q\|_{E} \\
& \leq\left(1+\frac{2 \theta_{2} \rho^{m}}{1-\theta_{2} \rho^{m}} \cdot \frac{d \delta^{\mu}}{\theta_{3}^{\mu}}\right)\left(1+\theta_{1} \delta^{\mu}\right)^{d(m+1)}\|Q\|_{E} \quad\left(\text { since } \delta>\theta_{3} d^{-1 / \mu}\right) \\
& \leq\left(1+d \theta_{6} \delta^{\mu}\right)\left(1+\theta_{1} \delta^{\mu}\right)^{d(m+1)}\|Q\|_{E} \\
& \leq\left(1+\theta_{6} \delta^{\mu}\right)^{d}\left(1+\theta_{1} \delta^{\mu}\right)^{d(m+1)}\|Q\|_{E} \\
& \leq\left(1+\theta_{6} \delta^{\mu}\right)^{d(m+2)}\|Q\|_{E} \\
& \leq\left(1+\theta_{6} \theta_{7} \delta^{\mu}\right)^{d}\|Q\|_{E} \\
& \leq\left(1+\theta_{8} \delta^{\mu}\right)^{d}\|Q\|_{E} \text {, }
\end{aligned}
$$

which establishes the estimate (4.10).

To complete the proof, note that $h(E)$ has the HCP property. Indeed, since $\hat{E} \subset U_{*}$ (see (4.3)) and $E$ has the HCP property (see (4.9)), we can apply Theorem 1.9 to the set $E$ and the map $\left.h\right|_{U_{*}}$. Therefore, there exist $\theta, \gamma>0$ such that, for all $y, y^{\prime} \in \mathbb{C}^{N^{\prime}}$,

$$
\left|V_{h(E)}(y)-V_{h(E)}\left(y^{\prime}\right)\right| \leq \theta\left|y-y^{\prime}\right|^{\gamma} .
$$

Recall also that we are now working under the assumption that (iii) holds, and so

$$
h(E) \subset h(K) \subset h(E) .
$$

Consequently,

$$
V_{h(E)} \geq V_{h(K)} \geq V_{h(E)^{\Upsilon}}
$$

On the other hand, [23, Theorem 5.1.7] tell us that $V_{h(E)^{-}}=V_{h(E)}$. Therefore, $V_{h(K)}=$ $V_{h(E)}$. Together with (4.11), this completes the proof of (i) under the assumption that (iii) holds.

\section{Proof of Theorem 1.12}

Proof of Theorem 1.12 Take an open, bounded set $\Omega \subset \mathbb{C}^{N}$ such that $\hat{K} \subset \Omega$ and $\bar{\Omega} \subset U$. Since $h(K)$ is a nonpluripolar subset of $\mathbb{C}^{N^{\prime}}$ and $h(\Omega)$ is bounded,

$$
C_{1}:=\sup _{h(\Omega)} \Phi_{h(K)}<+\infty
$$


see (1.1) and (1.2).

As in the proof of Theorem 1.9, we show that there exist a set $E \subset \mathbb{C}^{N}$ and constants $C_{2}>0, \rho \in(0,1)$ such that

- $K \subset \operatorname{Int} E$ and $E \subset \Omega$,

- for each holomorphic and bounded function $f: \Omega \rightarrow \mathbb{C}$ and each $v \in \mathbb{N}$, there exists a polynomial $R_{v} \in \mathbb{C}\left[z_{1}, \ldots, z_{N}\right]$ with $\operatorname{deg} R_{v} \leq v$ and such that

$$
\left\|f-R_{v}\right\|_{E} \leq C_{2}\|f\|_{\Omega} \rho^{v}
$$

Furthermore, take $k \in \mathbb{N}$ and $t_{1}>0$ such that

$$
C_{1} \rho^{k} \leq 1
$$

and

$$
K_{\left(t_{1}\right)} \subset E
$$

Let $\varepsilon, C>0$ be of the definition of Markov's inequality for the set $\mathrm{K}$. That is, for each polynomial $R \in \mathbb{C}\left[z_{1}, \ldots, z_{N}\right]$ and each $\beta \in \mathbb{N}_{0}^{N}$,

$$
\left\|D^{\beta} R\right\|_{K} \leq\left(C(\operatorname{deg} R)^{\varepsilon}\right)^{|\beta|}\|R\|_{K} .
$$

Moreover, let $\varkappa, \theta, t_{*}>0$ and $q \in \mathbb{N}$ be of Theorem 1.13. Set

$$
\begin{aligned}
& \kappa:=\varkappa(2+\varepsilon), \\
& C_{3}:=\min \left\{t_{*}, t_{1}, \frac{1}{C k^{\varepsilon}}\right\}, \\
& C_{4}:=\sup _{j \in \mathbb{N}}\left(\frac{1+\frac{1}{\sqrt{2} j}}{1-\frac{1}{\sqrt{2} j}}\right)^{j q}, \\
& C_{5}:=\frac{\theta C_{3}^{\varkappa}}{2^{\varkappa+1}}, \\
& C_{6}:=C_{2} C_{4} 2^{N^{\prime}}+\left(C_{2}+1\right) C_{4} e^{N} 2^{N^{\prime}} .
\end{aligned}
$$

Fix a polynomial $P \in \mathbb{C}\left[y_{1}, \ldots, y_{N^{\prime}}\right]$ with $\operatorname{deg} P \leq n(n \in \mathbb{N})$, and also fix $y \in h(K)_{\left(C_{5} n^{-\kappa}\right)}$. We now show that

$$
|P(y)| \leq C_{6}\|P\|_{h(K)} .
$$

Set $l=l(n):=k n$. By (5.1), there exists a polynomial $R_{l} \in \mathbb{C}\left[z_{1}, \ldots, z_{N}\right]$ with $\operatorname{deg} R_{l} \leq l$ and such that

$$
\left\|P \circ h-R_{l}\right\|_{E} \leq C_{2}\|P \circ h\|_{\Omega} \rho^{l} .
$$


Note that

$$
\left\|P \circ h-R_{l}\right\|_{E} \leq C_{2}\|P\|_{h(K)} .
$$

Indeed,

$$
\begin{aligned}
\left\|P \circ h-R_{l}\right\|_{E} & \leq C_{2}\|P\|_{h(\Omega)} \rho^{l} \\
& \leq C_{2} \rho^{l}\left(\sup _{h(\Omega)} \Phi_{h(K)}\right)^{n}\|P\|_{h(K)} \\
& =C_{2}\left(C_{1} \rho^{k}\right)^{n}\|P\|_{h(K)} \\
& \leq C_{2}\|P\|_{h(K)}
\end{aligned}
$$

which proves (5.7).

Put $v_{n}:=C_{3} / n^{\varepsilon}$ and $\tau_{n}:=v_{n} / 2 n^{2}$. Take $a \in K$ such that $\operatorname{dist}(y, h(K))=$ $|y-h(a)|$. Note that $v_{n} \leq t_{1}$, and hence

$$
\mathbb{D}\left(a, v_{n}\right) \subset E
$$

see (5.3).

Let $Q_{a}: \mathbb{C} \rightarrow \mathbb{C}^{N^{\prime}}$ be the polynomial map of Theorem 1.13. In particular, $Q_{a}(0)=$ $h(a)$. Then

$$
\begin{aligned}
|P(y)| & =\left|\sum_{\alpha \in \mathbb{N}_{0}^{N^{\prime}}} \frac{D^{\alpha} P(h(a))}{\alpha !}(y-h(a))^{\alpha}\right| \\
& \leq \sum_{\alpha \in \mathbb{N}_{0}^{N^{\prime}}} \frac{\left|D^{\alpha} P\left(Q_{a}(0)\right)\right|}{\alpha !}\left(\frac{C_{5}}{n^{\kappa}}\right)^{|\alpha|} \\
& \leq \sum_{\alpha \in \mathbb{N}_{0}^{N^{\prime}}} \frac{\left\|D^{\alpha} P \circ Q_{a}\right\|_{\left[\tau_{n}, v_{n}\right]}}{\alpha !}\left(\Phi_{\left[\tau_{n}, v_{n}\right]}(0)\right)^{n q}\left(\frac{C_{5}}{n^{\kappa}}\right)^{|\alpha|} \\
& =\sum_{\alpha \in \mathbb{N}_{0}^{N^{\prime}}} \frac{\left\|D^{\alpha} P \circ Q_{a}\right\|_{\left[\tau_{n}, v_{n}\right]}}{\alpha !}\left(\frac{1+\frac{1}{\sqrt{2} n}}{1-\frac{1}{\sqrt{2} n}}\right)^{n q}\left(\frac{C_{5}}{n^{\kappa}}\right)^{|\alpha|} .
\end{aligned}
$$

Here we have used the following formula:

$$
\Phi_{[\tau, v]}(u)=\frac{1+\sqrt{\frac{\tau-u}{v-u}}}{\left|1-\sqrt{\frac{\tau-u}{v-u}}\right|},
$$


which is valid for all $\tau, v \in \mathbb{R}$ with $\tau<v$ and all $u \in(-\infty, \tau] \cup(v,+\infty)$; see for example [31, Lemma 2.1]. Consequently,

$$
|P(y)| \leq \sum_{\alpha \in \mathbb{N}_{0}^{N^{\prime}}} \frac{C_{4}}{\alpha !}\left|D^{\alpha} P \circ Q_{a}\left(s_{n}(\alpha)\right)\right|\left(\frac{C_{5}}{n^{\kappa}}\right)^{|\alpha|}
$$

for some $s_{n}(\alpha) \in\left[\tau_{n}, v_{n}\right]$.

Theorem 1.13 and the estimates $0<s_{n}(\alpha) \leq v_{n} \leq t_{*}$ imply that

$$
\mathbb{D}\left(Q_{a}\left(s_{n}(\alpha)\right), \theta s_{n}(\alpha)^{\varkappa}\right) \subset h\left(\mathbb{D}\left(a, v_{n}\right)\right)
$$

Therefore,

$$
\begin{aligned}
& |P(y)| \leq \sum_{\alpha \in \mathbb{N}_{0}^{N^{\prime}}} C_{4} \frac{\|P\|_{h\left(\mathbb{D}\left(a, v_{n}\right)\right)}}{\left(\theta s_{n}(\alpha)^{\varkappa}\right)^{|\alpha|}}\left(\frac{C_{5}}{n^{\kappa}}\right)^{|\alpha|} \quad \text { (by (5.9), (5.10), and Cauchy's ineq.) } \\
& \leq \sum_{\alpha \in \mathbb{N}_{0}^{N^{\prime}}} C_{4}\left(\frac{C_{5}}{\theta \tau_{n}^{\varkappa} n^{\kappa}}\right)^{|\alpha|}\|P\|_{h\left(\mathbb{D}\left(a, v_{n}\right)\right)} \\
& =\sum_{\alpha \in \mathbb{N}_{0}^{N^{\prime}}} \frac{C_{4}}{2^{|\alpha|}}\|P\|_{h\left(\mathbb{D}\left(a, v_{n}\right)\right)} \\
& =C_{4} 2^{N^{\prime}}\|P \circ h\|_{\mathbb{D}\left(a, v_{n}\right)} \\
& \leq C_{4} 2^{N^{\prime}}\left(\left\|P \circ h-R_{l}\right\|_{E}+\left\|R_{l}\right\|_{\mathbb{D}\left(a, v_{n}\right)}\right) \quad(\text { by }(5.8)) \text {. }
\end{aligned}
$$

Hence, by (5.7),

$$
|P(y)| \leq C_{4} 2^{N^{\prime}}\left(C_{2}\|P\|_{h(K)}+\left\|R_{l}\right\|_{\mathbb{D}\left(a, v_{n}\right)}\right) .
$$

Take $z_{0} \in \mathbb{D}\left(a, v_{n}\right)$ such that $\left|R_{l}\left(z_{0}\right)\right|=\left\|R_{l}\right\|_{\mathbb{D}\left(a, v_{n}\right)}$. Then

$$
\begin{aligned}
\left\|R_{l}\right\|_{\mathbb{D}\left(a, v_{n}\right)} & =\left|\sum_{\beta \in \mathbb{N}_{0}^{N}} \frac{D^{\beta} R_{l}(a)}{\beta !}\left(z_{0}-a\right)^{\beta}\right| \\
& \leq \sum_{\beta \in \mathbb{N}_{0}^{N}} \frac{\left|D^{\beta} R_{l}(a)\right|}{\beta !} v_{n}^{|\beta|} \\
& \leq \sum_{\beta \in \mathbb{N}_{0}^{N}} \frac{\left(C l^{\varepsilon}\right)^{|\beta|}\left\|R_{l}\right\|_{K}}{\beta !} v_{n}^{|\beta|} \\
& =\sum_{\beta \in \mathbb{N}_{0}^{N}} \frac{\left(C C_{3} k^{\varepsilon}\right)^{|\beta|}}{\beta !}\left\|R_{l}\right\|_{K}
\end{aligned}
$$




$$
\begin{aligned}
& \leq \sum_{\beta \in \mathbb{N}_{0}^{N}} \frac{\left\|R_{l}\right\|_{K}}{\beta !} \\
& =e^{N}\left\|R_{l}\right\|_{K} \\
& \leq e^{N}\left(\left\|P \circ h-R_{l}\right\|_{K}+\|P \circ h\|_{K}\right) \\
& \leq\left(C_{2}+1\right) e^{N}\|P\|_{h(K)}
\end{aligned}
$$

Combining this with (5.11), we get

$$
|P(y)| \leq C_{4} 2^{N^{\prime}}\left(C_{2}\|P\|_{h(K)}+\left(C_{2}+1\right) e^{N}\|P\|_{h(K)}\right)=C_{6}\|P\|_{h(K)},
$$

which establishes the estimate (5.5).

By the remark following Definition 1.10 and by $(5.5), h(K)$ satisfies Markov's inequality, which is the desired conclusion.

\section{A refinement of Theorems 1.9 and 1.12}

We conclude this paper with one more result, which is a slight strengthening of Theorems 1.9 and 1.12 .

Theorem 6.1 Let $h: U \rightarrow \mathbb{C}^{N^{\prime}}$, where $U \subset \mathbb{C}^{N}$ is an open set, be a nondegenerate holomorphic map $\left(N, N^{\prime} \in \mathbb{N}\right)$. Assume that $\mathcal{Z} \subset \mathbb{C}^{N}$ is a compact set with $\hat{\mathcal{Z}} \subset U$. Then there exist $\eta_{1}, \eta_{2}>0$ such that:

(1) For each compact set $\emptyset \neq K \subset \mathcal{Z}$ having the HCP property with the exponent $\mu>0$ (that is, (1.4) holds with some $\varpi>0$ ), the set $h(K)$ has the HCP property with the exponent $\eta_{1} \mu$.

(2) For each compact set $K \subset \mathcal{Z}$ satisfying Markov's inequality with the exponent $\varepsilon>0$ (that is, (1.5) holds with some $C>0$ ) and such that $h(K)$ is a nonpluripolar subset of $\mathbb{C}^{N^{\prime}}$, the set $h(K)$ satisfies Markov's inequality with the exponent $\eta_{2} \varepsilon$.

Proof Let $\varkappa>0$ be of Theorem 1.13 applied to $\mathcal{Z}$ (instead of $K$ ) and take $\tilde{\varkappa} \in \mathbb{N}$ such that $\tilde{\varkappa} \geq \varkappa$. Set $\eta_{1}:=1 /(3 \tilde{\varkappa})$ and $\eta_{2}:=3 \varkappa$. Fix a compact set $\emptyset \neq K \subset \mathcal{Z}$. Note that $\hat{K} \subset \hat{\mathcal{Z}} \subset U$. Put

$$
r:=\sup \left\{\left|z_{1}\right|:\left(z_{1}, \ldots, z_{N}\right) \in K\right\}
$$

and take $a \in K$ such that $\left|a_{1}\right|=r$. If $r>0$, then for each $n \in \mathbb{N}$, let $Q_{n} \in$ $\mathbb{C}\left[z_{1}, \ldots, z_{N}\right]$ be defined by $Q_{n}(z):=\left(z_{1} / r\right)^{n}$. Clearly, $\left\|Q_{n}\right\|_{K}=1$. Note that $K$ being a Markov set implies $r>0$. This is immediately seen by considering the polynomial $Q(z):=z_{1}$.

Assume first that $K$ has the HCP property with the exponent $\mu>0$, that is, there exists $\varpi>0$ such that, for each $z \in K_{(1)}$,

$$
V_{K}(z) \leq \varpi(\operatorname{dist}(z, K))^{\mu}
$$


Then $r>0$ and, for each $n \in \mathbb{N}$, we have from (1.2), (1.3) and (6.1):

$$
\begin{aligned}
\frac{n}{r} & <n^{1 / \mu}\left(1+\frac{1}{n^{1 / \mu} r}\right)^{n}=n^{1 / \mu}\left\|Q_{n}\right\|_{\mathbb{D}\left(a, n^{-1 / \mu}\right)} \\
& \leq n^{1 / \mu}\left(\sup _{\mathbb{D}\left(a, n^{-1 / \mu}\right)} \Phi_{K}\right)^{n}\left\|Q_{n}\right\|_{K} \leq n^{1 / \mu} e^{\varpi} .
\end{aligned}
$$

Thus, $n / r \leq n^{1 / \mu} e^{\varpi}$, which implies $\mu \leq 1$; see also [46, Remark 3.7]. Analysis of the proof of Theorem 1.9 shows that the set $h(K)$ has the HCP property with the exponent $\mu /(\tilde{\varkappa}(2 \mu+1))$. However, $\mu /(\tilde{\varkappa}(2 \mu+1)) \geq \mu /(3 \tilde{\varkappa})=\eta_{1} \mu$. Therefore, $h(K)$ has the HCP property with the exponent $\eta_{1} \mu$, and (1) is proved.

In order to prove (2), assume that $h(K)$ is a nonpluripolar subset of $\mathbb{C}^{N^{\prime}}$ and $K$ satisfies Markov's inequality with the exponent $\varepsilon>0$, that is, there exists $C>0$ such that, for each polynomial $Q \in \mathbb{C}\left[z_{1}, \ldots, z_{N}\right]$ and each $\alpha \in \mathbb{N}_{0}^{N}$,

$$
\left\|D^{\alpha} Q\right\|_{K} \leq\left(C(\operatorname{deg} Q)^{\varepsilon}\right)^{|\alpha|}\|Q\|_{K}
$$

Then $r>0$ and, for each $n \in \mathbb{N}$, we have from (6.2):

$$
\frac{n}{r}=\left|\frac{\partial Q_{n}}{\partial z_{1}}(a)\right| \leq C n^{\varepsilon}\left\|Q_{n}\right\|_{K}=C n^{\varepsilon} .
$$

It follows that $n / r \leq C n^{\varepsilon}$, which implies $\varepsilon \geq 1$. A careful look at the proof of Theorem 1.12 reveals that the set $h(K)$ satisfies Markov's inequality with the exponent $\varkappa(2+\varepsilon)$. But $\varkappa(2+\varepsilon) \leq 3 \varkappa \varepsilon=\eta_{2} \varepsilon$. Consequently, $h(K)$ satisfies Markov's inequality with the exponent $\eta_{2} \varepsilon$, which proves (2).

Acknowledgements The author is grateful to the referee for the comments and suggestions, which improved the exposition. The research was partially supported by the NCN grant 2015/17/B/ST1/00614.

Open Access This article is licensed under a Creative Commons Attribution 4.0 International License, which permits use, sharing, adaptation, distribution and reproduction in any medium or format, as long as you give appropriate credit to the original author(s) and the source, provide a link to the Creative Commons licence, and indicate if changes were made. The images or other third party material in this article are included in the article's Creative Commons licence, unless indicated otherwise in a credit line to the material. If material is not included in the article's Creative Commons licence and your intended use is not permitted by statutory regulation or exceeds the permitted use, you will need to obtain permission directly from the copyright holder. To view a copy of this licence, visit http://creativecommons.org/licenses/by/4.0/.

\section{References}

1. Baran, M., Białas-Cież, L.: Hölder continuity of the Green function and Markov brothers' inequality. Constr. Approx. 40, 121-140 (2014)

2. Baran, M., Białas-Cież, L., Milówka, B.: On the best exponent in Markov inequality. Potential Anal. 38, 635-651 (2013)

3. Baran, M., Pleśniak, W.: Markov's exponent of compact sets in $\mathbb{C}^{n}$. Proc. Am. Math. Soc. 123, 27852791 (1995) 
4. Bedford, E., Taylor, B.A.: A new capacity for plurisubharmonic functions. Acta Math. 149, 1-40 (1982)

5. Bierstone, E., Milman, P.: Semianalytic and subanalytic sets. Inst. Hautes Études Sci. Publ. Math. 67, 5-42 (1988)

6. Borwein, P., Erdélyi, T.: Polynomials and polynomial inequalities. Springer-Verlag, New York (1995)

7. Bos, L.P., Brudnyi, A., Levenberg, N.: On polynomial inequalities on exponential curves in $\mathbb{C}^{n}$. Constr. Approx. 31, 139-147 (2010)

8. Bos, L.P., Brudnyi, A., Levenberg, N., Totik, V.: Tangential Markov inequalities on transcendental curves. Constr. Approx. 19, 339-354 (2003)

9. Bos, L., Levenberg, N., Milman, P.D., Taylor, B.A.: Tangential Markov inequalities characterize algebraic submanifolds of $\mathbb{R}^{N}$. Indiana Univ. Math. J. 44, 115-138 (1995)

10. Bos, L., Levenberg, N., Milman, P.D., Taylor, B.A.: Tangential Markov inequalities on real algebraic varieties. Indiana Univ. Math. J. 47, 1257-1272 (1998)

11. Bos, L.P., Milman, P.D.: On Markov and Sobolev type inequalities on sets in $\mathbb{R}^{n}$. In: Rassias, Th.M., Srivastava, H.M., Yanushauskas, A. (eds.) Topics in polynomials of one and several variables and their applications, pp. 81-100. World Sci., Singapore (1993)

12. Bos, L.P., Milman, P.D.: Sobolev-Gagliardo-Nirenberg and Markov type inequalities on subanalytic domains. Geom. Funct. Anal. 5, 853-923 (1995)

13. Bos, L.P., Milman, P.D.: Tangential Markov inequalities on singular varieties. Indiana Univ. Math. J. 55, 65-73 (2006)

14. Brudnyi, A.: Local inequalities for plurisubharmonic functions. Ann. Math. 149, 511-533 (1999)

15. Brudnyi, A.: On local behavior of holomorphic functions along complex submanifolds of $\mathbb{C}^{N}$. Invent. Math. 173, 315-363 (2008)

16. Carleson, L., Totik, V.: Hölder continuity of Green's functions. Acta Sci. Math. 70, 557-608 (2004)

17. Cartan, H.: Differential calculus. Hermann, Paris (1971)

18. van den Dries, L.: Tame topology and o-minimal structures, LMS Lecture Note Series, vol. 248. Cambridge University Press, Cambridge (1998)

19. Erdélyi, T., Kroó, A.: Markov-type inequalities on certain irrational arcs and domains. J. Approx. Theory 130, 111-122 (2004)

20. Frerick, L.: Extension operators for spaces of infinite differentiable Whitney jets. J. Reine Angew. Math. 602, 123-154 (2007)

21. Goncharov, A.: A compact set without Markov's property but with an extension operator for $\mathcal{C}^{\infty}$ functions. Studia Math. 119, 27-35 (1996)

22. Hörmander, L.: An introduction to complex analysis in several variables. North-Holland, Amsterdam (1990)

23. Klimek, M.: Pluripotential theory. Oxford University Press, Oxford (1991)

24. Kroó, A., Szabados, J.: Markov-Bernstein type inequalities for multivariate polynomials on sets with cusps. J. Approx. Theory 102, 72-95 (2000)

25. Levenberg, N.: Approximation in $\mathbb{C}^{N}$. Surv. Approx. Theory 2, 92-140 (2006)

26. Levenberg, N.: Ten lectures on weighted pluripotential theory. Dolomit. Res. Notes. Approx. 5, 1-59 (2012)

27. Łojasiewicz, S.: Ensembles semi-analytiques, Lecture Notes. IHES, Bures-sur-Yvette, France (1965)

28. Łojasiewicz, S.: Introduction to complex analytic geometry. Birkhäuser Verlag, Basel (1991)

29. Pawłucki, W., Pleśniak, W.: Markov's inequality and $\mathcal{C}^{\infty}$ functions on sets with polynomial cusps. Math. Ann. 275, 467-480 (1986)

30. Pierzchała, R.: UPC condition in polynomially bounded o-minimal structures. J. Approx. Theory 132, 25-33 (2005)

31. Pierzchała, R.: Siciak’s extremal function of non-UPC cusps. I. J. Math. Pures Appl. 94, 451-469 (2010)

32. Pierzchała, R.: Markov's inequality in the o-minimal structure of convergent generalized power series. Adv. Geom. 12, 647-664 (2012)

33. Pierzchała, R.: On the Bernstein-Walsh-Siciak theorem. Studia Math. 212(1), 55-63 (2012)

34. Pierzchała, R.: Remez-type inequality on sets with cusps. Adv. Math. 281, 508-552 (2015)

35. Pierzchała, R.: Markov's inequality and polynomial mappings. Math. Ann. 366, 57-82 (2016)

36. Pleśniak, W.: Invariance of the $L$-regularity of compact sets in $\mathbb{C}^{N}$ under holomorphic mappings. Trans. Amer. Math. Soc. 246, 373-383 (1978)

37. Pleśniak, W.: Compact subsets of $\mathbb{C}^{n}$ preserving Markov's inequality. Mat. Vesnik 40, 295-300 (1988) 
38. Pleśniak, W.: Markov's inequality and the existence of an extension operator for $\mathcal{C}^{\infty}$ functions. J. Approx. Theory 61, 106-117 (1990)

39. Pleśniak, W.: Siciak's extremal function in complex and real analysis. Ann. Polon. Math. 80, 37-46 (2003)

40. Pleśniak, W.: Inégalité de Markov en plusieurs variables. Internat. J. Math. Math. Sci. 1-12, (2006)

41. Pleśniak, W.: Multivariate Jackson inequality. J. Comput. Appl. Math. 233, 815-820 (2009)

42. Sadullaev, A., Zeriahi, A.: Hölder regularity of generic manifolds. Ann. Sc. Norm. Super. Pisa Cl. Sci. (5) 16, 369-382 (2016)

43. Siciak, J.: On some extremal functions and their applications in the theory of analytic functions of several complex variables. Trans. Amer. Math. Soc. 105, 322-357 (1962)

44. Siciak, J.: Extremal plurisubharmonic functions in $\mathbb{C}^{n}$. Ann. Polon. Math. 39, 175-211 (1981)

45. Siciak, J.: Rapid polynomial approximation on compact sets in $\mathbb{C}^{N}$. Univ. Iagel. Acta Math. 30, 145154 (1993)

46. Siciak, J.: Wiener's type sufficient conditions in $\mathbb{C}^{N}$. Univ. Iagel. Acta Math. 35, 47-74 (1997)

47. Totik, V.: Polynomial inverse images and polynomial inequalities. Acta Math. 187, 139-160 (2001)

Publisher's Note Springer Nature remains neutral with regard to jurisdictional claims in published maps and institutional affiliations. 